\title{
Hilbert space renormalization for the many-electron problem
}

Zhendong Li and Garnet Kin-Lic Chan

Citation: J. Chem. Phys. 144, 084103 (2016); doi: 10.1063/1.4942174

View online: http://dx.doi.org/10.1063/1.4942174

View Table of Contents: http://aip.scitation.org/toc/jcp/144/8

Published by the American Institute of Physics 


\title{
Hilbert space renormalization for the many-electron problem
}

\author{
Zhendong $\mathrm{Li}^{\mathrm{a})}$ and Garnet Kin-Lic Chan ${ }^{\mathrm{b})}$ \\ Department of Chemistry, Frick Laboratory, Princeton University, Princeton, New Jersey 08544, USA
}

(Received 15 December 2015; accepted 4 February 2016; published online 23 February 2016)

\begin{abstract}
Renormalization is a powerful concept in the many-body problem. Inspired by the highly successful density matrix renormalization group (DMRG) algorithm, and the quantum chemical graphical representation of configuration space, we introduce a new theoretical tool: Hilbert space renormalization, to describe many-electron correlations. While in DMRG, the many-body states in nested Fock subspaces are successively renormalized, in Hilbert space renormalization, many-body states in nested Hilbert subspaces undergo renormalization. This provides a new way to classify and combine configurations. The underlying wavefunction Ansatz, namely, the Hilbert space matrix product state (HS-MPS), has a very rich and flexible mathematical structure. It provides low-rank tensor approximations to any configuration interaction (CI) space through restricting either the "physical indices" or the coupling rules in the HS-MPS. Alternatively, simply truncating the "virtual dimension" of the HS-MPS leads to a family of size-extensive wave function Ansätze that can be used efficiently in variational calculations. We make formal and numerical comparisons between the HS-MPS, the traditional Fock-space MPS used in DMRG, and traditional CI approximations. The analysis and results shed light on fundamental aspects of the efficient representation of manyelectron wavefunctions through the renormalization of many-body states. (C) 2016 AIP Publishing LLC. [http://dx.doi.org/10.1063/1.4942174]
\end{abstract}

\section{INTRODUCTION}

Accurate and efficient solutions of the many-electron problem are central to all aspects of chemistry and physics. ${ }^{1}$ After many decades of investigation, ${ }^{2}$ it has been common to distinguish two kinds of many-electron correlation: static or strong correlation, associated with the qualitative physics of the valence orbitals; and dynamic correlation, arising from Coulomb interactions at short range, and associated with a very large number of high-energy degrees of freedom.

Configuration interaction (CI), ${ }^{3}$ many-body perturbation theory, and coupled cluster theory, ${ }^{4}$ as well as explicit correlation techniques, ${ }^{5-7}$ have traditionally presented highly successful approaches to describe dynamic correlation. Recent advances in tensor network states (TNS) ${ }^{8-10}$ from condensed matter physics and quantum information theory, have further led to efficient representations of strong correlation also. The most widely used example of this latter class is the density matrix renormalization group (DMRG) method, ${ }^{11-14}$ which employs the simple one-dimensional TNS, viz., the matrix product state (MPS). ${ }^{15}$ DMRG has been successfully applied in quantum chemistry to compute near-exact many-electron wavefunctions of several systems with a very large number of valence quantum degrees of freedom, ${ }^{10,16-23}$ such as the oxygen-evolving complex $\left(\mathrm{Mn}_{4} \mathrm{CaO}_{5}\right)^{24}$ and the iron-sulfur clusters. ${ }^{25}$

Successfully combining these different representations for static and dynamic correlation is a nontrivial problem, and is an important research challenge. ${ }^{26-30}$ For example,

\footnotetext{
a)Electronic mail: zhendong@ princeton.edu

b)Electronic mail: gkchan@ princeton.edu
}

TNS methods and dynamic correlation methods rely on very different physical pictures. TNS approximations parametrize the entanglement between groups of orbitals (referred to as sites), and the variational objects are states in the local Fock subspaces of the orbitals, which span the complete set of particle numbers in each subspace. In contrast, traditional dynamic correlation methods are expressed in terms of particle-hole excitations relative to a given $N$-electron reference. The variational objects or amplitudes are then of fixed excitation rank, and act on a given number of particles and holes at a time. Bridging these two very different parametrizations is a challenge in developing new theories for the electron correlation problem.

To this end, we consider here the possibility to formulate a new kind of TNS. Unlike existing TNS, it will be expressed in a set of nested Hilbert, as opposed to Fock, subspaces. The resulting wavefunction is an $N$-electron wavefunction directly in Hilbert space, rather than in the Fock-space (occupation number) representation. In essence, this means that the "sites" in the TNS will refer to electrons rather than orbitals, and the $N$-electron wavefunction will be expressed by an interconnected network of $N$ such sites. At first glance, this does not seem natural, as fermion antisymmetry precludes a simple factorization of $N$-electron wavefunctions into a product of single-electron wavefunctions. Indeed, one might suspect that imposing antisymmetry could mean that the cost of working with a Hilbert space TNS will be factorial with respect to the number of electrons $N$. As an example, the antisymmetrized product of geminals (APG) ${ }^{31-33}$ wavefunction can be considered to be a simple Hilbert space TNS where electrons (sites) within pairs are connected by pair expansion coefficients. However, even such a formally 
simple Ansatz is computationally intractable, unless strong orthogonality constraints are imposed between the geminals. Indeed, fermion antisymmetry is an important reason why it is easier to formulate TNS in Fock space, where the antisymmetry is handled by the anticommuting operator algebra, leaving a simple direct product structure in the state space.

In this work, we will show, however, that the above difficulty of antisymmetry can be circumvented by introducing "prefix" and "suffix" constraints for renormalizations in Hilbert space. This enables a simple composition of two renormalized states, and more importantly the efficient computation of operator matrix elements, such as for the overlap or Hamiltonian. This allows practical algorithms, such as the variational optimization of wavefunction parameters, to be formulated and applied. The layout of the remainder of the paper is as follows: The basic concepts of Fock-space MPS (FS-MPS) are recapitulated in Sec. II, where the important connection with the graphical representation of configuration space $^{34}$ is also made. An alternative view of the same graph naturally leads to the Hilbert space MPS (HS-MPS) and the necessary prefix/suffix constraints for renormalizations in Hilbert space to make the HS-MPS computationally tractable. The detailed mathematical formulation of the HS-MPS is presented in Sec. III, along with formal comparisons with FS-MPS and CI (Secs. III A-III E). We further describe the DMRG-like algorithm for the variational optimization of HS-MPS (Secs. III F and III G), the generalizations to bosons and spin-adaptation of the HS-MPS (Sec. III H), and connections with other theories (Sec. III I) including the factorization of multivariate polynomials, artificial neural networks (ANNs), and the graphically contracted function (GCF) method of Shepard et al. ${ }^{35-39}$ Numerical studies for several typical chemical systems are presented in Sec. IV, aiming at a numerical comparison of HS-MPS and FS-MPS. Conclusions are given in Sec. V.

\section{FS-MPS AND ITS GRAPHICAL REPRESENTATION}

Any $N$-electron wavefunction $|\Psi\rangle$ can be written in the Fock-space (occupation-number) representation ${ }^{2}$ as,

$$
|\Psi\rangle=\sum_{n_{1} n_{2} \cdots n_{K}} \Psi^{n_{1} n_{2} \cdots n_{K}}\left|n_{1} n_{2} \cdots n_{K}\right\rangle,
$$

where $n_{k} \in\{0,1\}$ is the occupation number for the $k$-th spin-orbital and $K$ is the total number of spin-orbitals. The coefficients $\Psi^{n_{1} n_{2} \cdots n_{K}} \in \mathbb{C}^{2^{K}}$ form a (complex) tensor of dimension $2^{K}$, which has the structure

$$
\Psi^{n_{1} n_{2} \cdots n_{K}}= \begin{cases}\Psi^{n_{1} n_{2} \cdots n_{K}}, & \sum_{k=1}^{K} n_{k}=N, \\ 0, & \text { otherwise }\end{cases}
$$

in order to be a well-defined $N$-electron wavefunction. In the Fock-space representation, the system with $K$ orbitals can be viewed as a spin lattice with $K$ distinguishable sites. The FS-MPS (with open-boundary conditions) can be expressed as a chain of tensor products

$$
\Psi^{n_{1} n_{2} \cdots n_{K}}=\sum_{\left\{\alpha_{k}\right\}} A_{\alpha_{1}}^{n_{1}}[1] A_{\alpha_{1} \alpha_{2}}^{n_{2}}[2] \cdots A_{\alpha_{K-1}}^{n_{K}}[K],
$$

where $A_{\alpha_{k-1} \alpha_{k}}^{n_{k}}[k](1<k<K)$ is a rank-3 tensor, and the tensors at the boundary ( $k=1$ and $k=K$ ) are rank-2 tensors (i.e., matrices). For simplicity, we will use $A[k]$ to denote both kinds of tensors in the following discussion. In MPS terminology, the occupation number $n_{k}$ is usually referred as the "physical index," while $\alpha_{k}$ is referred as the "virtual index." The virtual index can be viewed to arise from successive singular value decompositions (SVD) of the Fock-space tensor $\Psi^{n_{1} n_{2} \cdots n_{K}}$, i.e.,

$$
\begin{aligned}
\Psi^{n_{1} n_{2} \cdots n_{K}} & =\sum_{\alpha_{1}} U_{\alpha_{1}}^{n_{1}} s_{\alpha_{1}} V_{\alpha_{1}}^{n_{2} n_{3} \cdots n_{K}} \triangleq \sum_{\alpha_{1}} U_{\alpha_{1}}^{n_{1}} W_{\alpha_{1}}^{n_{2} n_{3} \cdots n_{K}} \\
& =\sum_{\alpha_{1} \alpha_{2}} U_{\alpha_{1}}^{n_{1}} U_{\alpha_{1} \alpha_{2}}^{n_{2}} W_{\alpha_{2}}^{n_{3} \cdots n_{K}}=\cdots .
\end{aligned}
$$

The dimension of $\alpha_{k}$ denoted by $D_{k}$ is called "bond dimension" for the $k$-th "bond" between sites $k$ and $k+1$. The FS-MPS representation (3) can be exact as long as $D_{k}$ is sufficiently large. Given $K, N$, and a maximal level of CI excitations, the minimal values of $D_{k}(1 \leq k \leq K-1)$ for the exact representation of an arbitrary wavefunction in the specified configuration space can be determined, see Sec. III C. For convenience, we refer these minimal values as the "theoretical" bond dimensions. The minimal theoretical bond dimensions along the MPS chain increase exponentially for FCI, as $N$ and $K$ increase. The success of TNS lies in the fact that physically relevant states live only in a corner of the full configuration space, where the entanglement of the states is generally limited by the so-called "area law." 40,41 This implies that if the wavefunction on the right hand side of Eq. (3) with a finite bond dimension $D$ is taken as an approximate variational Ansatz, then to represent physically relevant states $D$ needs only to grow as polynomial function of $K$ and $N$ to achieve good accuracy. Notably, for ground states of one-dimensional gapped systems with Hamiltonians containing only local interactions, constant $D$ is sufficient to obtain accurate results for very large $N$ and $K$. This is the underlying reason why the DMRG algorithm works extremely well in one-dimensional systems.

The MPS representation (3) has a rich mathematical structure. One of the most significant, is that it naturally encodes a recursive chain of renormalization transformations. To illustrate this, the wavefunction (3) can be re-expressed in terms of the following renormalized intermediate states,

$$
\begin{aligned}
\left|\alpha_{1}\right\rangle & =\sum_{n_{1}}\left|n_{1}\right\rangle A_{\alpha_{1}}^{n_{1}}[1], \\
\left|\alpha_{2}\right\rangle & =\sum_{\alpha_{1} n_{2}}\left|\alpha_{1} n_{2}\right\rangle A_{\alpha_{1} \alpha_{2}}^{n_{2}}[2], \\
\left|\alpha_{K-1}\right\rangle & =\sum_{\alpha_{K-2^{n}} n_{K-1}}\left|\alpha_{K-2} n_{K-1}\right\rangle A_{\alpha_{K-2} \alpha_{K-1}}^{n_{K-1}}[K-1], \\
\left|\alpha_{K}\right\rangle & =\sum_{\alpha_{K-1} n_{K}}\left|\alpha_{K-1} n_{K}\right\rangle A_{\alpha_{K-1}}^{n_{K}}[K] \equiv|\Psi\rangle .
\end{aligned}
$$


It is now evident that the set of intermediate states $\left\{\left|\alpha_{k}\right\rangle\right\}$ are many-body states in the Fock space $\mathcal{F}_{k}$ defined by the direct product space of the first $k$ orbitals. From Eq. (5), the tensor $A[k]$ can be recognized as a linear map from the space spanned by renormalized states to the direct product space, e.g.,

$$
A[k]: \operatorname{span}\left\{\left|\alpha_{k}\right\rangle\right\} \mapsto \operatorname{span}\left\{\left|\alpha_{k-1}\right\rangle\right\} \otimes \operatorname{span}\left\{\left|n_{k}\right\rangle\right\} .
$$

From this perspective, this mapping is a many-body analog of the contraction of basis functions at the one-particle level, ${ }^{42}$ which maps a large underlying set of primitives to a smaller set of contracted functions. Without loss of generality, the set of renormalized states in Eq. (5) can be made orthonormal. This orthonormal set will be denoted by $\left\{\left|l_{k}\right\rangle\right\}$ and the corresponding MPS is then usually referred to as being in "left canonical form." ${ }^{14,19}$ Likewise, the renormalized intermediate states can be defined in "right canonical form" denoted by $\left\{\left|r_{k}\right\rangle\right\}$, where the renormalization process starts from the last site and proceeds to the first site. The FS-MPS can also be expressed in a basis of mixed forms, e.g.,

$$
\begin{aligned}
|\Psi\rangle & =\sum_{l_{k-1} n_{k} r_{k}}\left|l_{k-1} n_{k} r_{k}\right\rangle A_{l_{k-1} r_{k}}^{n_{k}}[k], \\
\left|l_{k-1}\right\rangle & =\sum_{n_{1} \cdots n_{k-1}}\left(A^{n_{1}}[1] \cdots A^{n_{k-1}}[k-1]\right)_{l_{k-1}}\left|n_{1} \cdots n_{k-1}\right\rangle \\
& =\sum_{l_{k-2} n_{k-1}}\left|l_{k-2} n_{k-1}\right\rangle A_{l_{k-2} n_{k-1}}^{n_{k-1}}[k-1], \\
\left|r_{k}\right\rangle & =\sum_{n_{k+1} \cdots n_{K}}\left(A^{n_{k+1}}[k+1] \cdots A^{n_{K}}[K]\right)_{r_{k}}\left|n_{k+1} \cdots n_{K}\right\rangle \\
& =\sum_{n_{k+1} r_{k+1}} A_{r_{k} r_{k+1}}^{n_{k+1}}[k+1]\left|n_{k+1} r_{k+1}\right\rangle .
\end{aligned}
$$

Such a mixed canonical form is particularly useful in numerical optimizations of MPS, since the basis $\left\{\left|l_{k-1} n_{k} r_{k}\right\rangle\right\}$ is orthonormal for each $k$.

For FS-MPS with particle number symmetry, by which we mean that the intermediate states $\left\{\left|\alpha_{k}\right\rangle\right\}$ are required to be eigenfunctions of particle number operators $\mathcal{N}_{k}$ $\triangleq \sum_{l=1}^{k} a_{l}^{\dagger} a_{l}$, the above recursive structure of FS-MPS can be visualized with the help of the graphical representation of the configuration space of determinants. ${ }^{34}$ The similar graphical representation in terms of configuration state functions rather than determinants, viz., the Shavitt graph in the Graphical Unitary Group Approach (GUGA) ${ }^{43-45}$ for CI, has also been used to motivate the construction of the graphical contracted function by Shepard and coworkers, ${ }^{37}$ a kind of FS-MPS as discussed in Sec. III I, although without the renormalization interpretation taken here. Figure 1(a) illustrates the configuration graph for $(K, N)=(6,4)$. Such graph is usually employed in CI algorithms, where any Slater determinant can be assigned a unique address based on the path from the origin $(0,0)$ to the destination $(K, N)$ on the graph. We note that the construction of such a graph is very much the same as the recursive construction in Eq. (5). For instance, starting from the origin, which is the vacuum (with no orbitals and no electron) the first orbital can be added to create new states. Depending on whether it is occupied $\left(n_{1}=1\right)$ or unoccupied $\left(n_{1}=0\right)$, either a new state, shown in the circle $(K, N)=(1,1)$, can be created, or the state remains

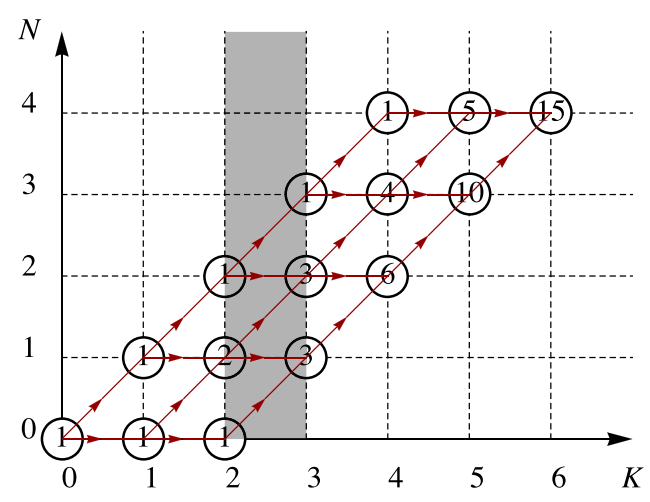

(a)

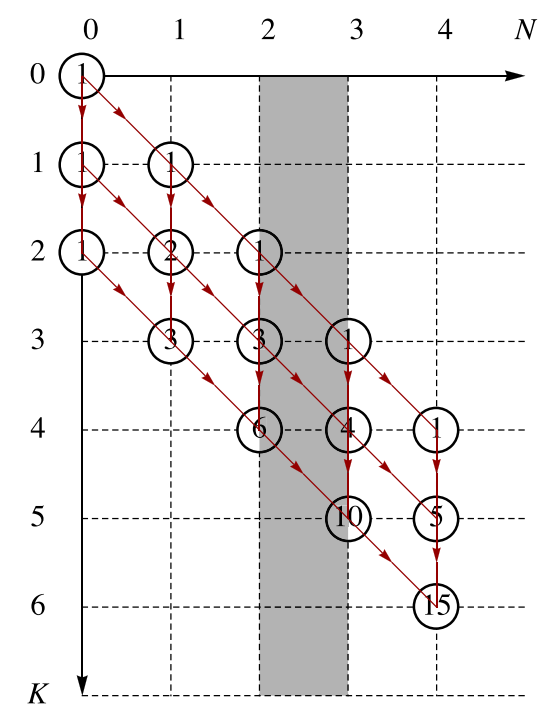

(b)

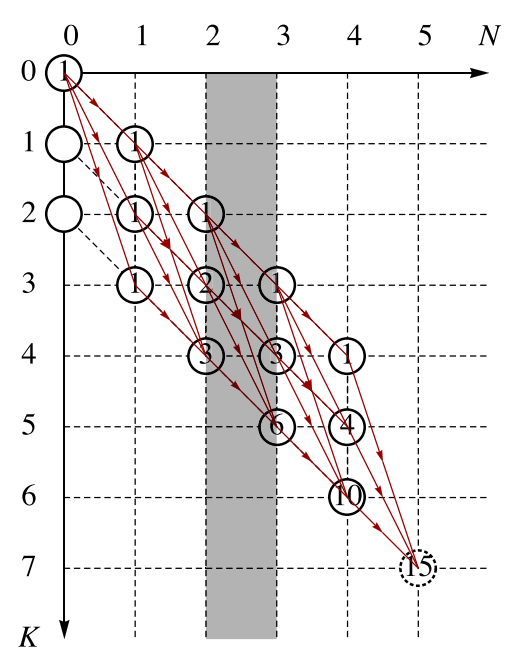

(c)

FIG. 1. Graphical representation of configuration space and renormalizations: (a) Configuration graph for $(K, N)=(6,4)$. (b) Rotated from (a) by $90^{\circ}$. (c) Readjusted configuration graph obtained by replacing all the downward arrows in (b). The shaded regions correspond to the tensor in Eq. (6).

a vacuum state $(K, N)=(1,0)$ in the Fock space $\mathcal{F}_{1}$. Thus, the two circles in the layer with $K=1$ comprise the Fock space $\mathcal{F}_{1}$. The stepwise construction can proceed until the last orbital $K$ is reached, which creates the whole Fock space. Therefore, the circle in the configuration graph with 
coordinate $(K, N)$ represents the Hilbert space $\mathcal{H}_{N}$, and the value in the circle shows the dimension of this space, given by the binomial coefficient $C_{K}^{N}$. The set of all circles in the same layer $K$ comprise the Fock space $\mathcal{F}_{K}=\bigoplus_{N=0}^{2^{K}} \mathcal{H}_{N}$. If only the states in the sector $(K, N)$ are of interest, then there is a parallelogram that restricts the possible intermediate states, see Figure 1(a). States outside of this parallelogram are irrelevant to the study of the target sector. From the same kind of recursive construction, one can immediately recognize that $A[k]$ (6) is the mapping from states in one layer to states in another layer, and its information is all contained in the shaded region between two layers if no truncation is made, e.g., see Figure 1(a) for $k=3$. Whenever there is a truncation in the renormalization process, the number of states in the corresponding circle that can enter the next layer gets reduced, for example, so as to avoid the exponential growth in the number of configurations. The ability to constrain the growth of configuration space is the very reason why renormalization is so powerful when combined with the DMRG algorithm to variationally optimize the intermediate states in Eq. (5), or equivalently the contraction coefficients in Eq. (6).

\section{HILBERT SPACE MPS}

\section{A. Formulation}

Having established the connection between FS-MPS and the graphical representation of configuration space, one may naturally wonder what is obtained if the renormalization is performed along the axis of particle numbers instead of orbitals. For this purpose, we can obtain a different view of Figure 1(a) by rotating it to Figure 1(b). (This is actually the more usual way to draw the configuration graph. ${ }^{34}$ ) We can then apply the same interpretation to the shaded region as a renormalization process.

It is easily identified that states in the same layer are now within the same $N$-electron Hilbert space. However, there is a crucial difference between Figures 1(a) and 1(b): While in the former case circles in the same layer represent different Hilbert subspaces of a Fock space, in the latter case, downward arrows between circles in the same layer indicate that the space of the circle above is a subspace of the space of the circle below, e.g., $\mathcal{H}_{n}^{k=n} \subset \mathcal{H}_{n}^{k=n+1} \subset \cdots \subset \mathcal{H}_{n}^{k=n+K-N}$ for the circles in the $n$th layer with different $k$ values. This makes the interpretation of Figure 1(b) as a renormalization flow less transparent. To avoid such difficulties, we write a slightly different graph, where the spaces associated with circles in the same layer are once again distinct, and obtained by taking the complementary part of the space to the circles above in Figure 1(b). That is, from Figure 1(b), each circle except the top one in the layer is replaced by the complementary part $\mathcal{P}_{n}^{k=p}$ $\left(\mathcal{H}_{n}^{k=p+1}=\mathcal{H}_{n}^{k=p} \oplus \mathcal{P}_{n}^{k=p}\right)$, such that the direct sum of all the circles gives rise to $\mathcal{H}_{n}^{k=n+K-N}=\bigoplus_{p=n}^{n+K-N} \mathcal{P}_{n}^{k=p}$. Note that $\mathcal{P}_{n}^{k=p}$ is nothing but the space spanned by those configurations that come from the previous layers, by occupying the $p$ th orbital as indicated by the southeasterly arrows in Figure 1(b). Thus, the dimension of these spaces can be read off from the previous layer in Figure 1(b). The changes are summarized in Figure 1(c), where the downward arrows have been eliminated and new oblique arrows with different slopes appear. The ending point $(k, n)$ of an arrow indicates that the $k$ th orbital is added to the $(n-1)$-electron states from the starting point of the arrow, to form new $n$-electron configurations that share the same last orbital $k$ (suffix) in their orbital string. Note that a virtual node at $(K+1, N+1)$ (dashed circle) has been added in Figure 1(c) to accommodate this new convention.

Applying the renormalization interpretation to Figure 1(c) now leads to a renormalization process that combines $n$ electron configurations that share the same orbital suffix. Similarly, in reverse, if the renormalization proceeds from the virtual node $(K+1, N+1)$ to the node $(0,0)$, then $n$ electron configurations that share the same orbital prefix will be combined. We refer to these renormalizations along the axis of particle numbers simply as Hilbert space renormalizations. Similar to Eq. (7), the left and right renormalized states can be defined recursively as

$$
\begin{aligned}
& |\Psi\rangle=\sum_{l_{i-1} p_{i} r_{i}}\left|l_{i-1} p_{i} r_{i}\right\rangle A_{l_{i-1} r_{i}}^{p_{i}}[i],
\end{aligned}
$$

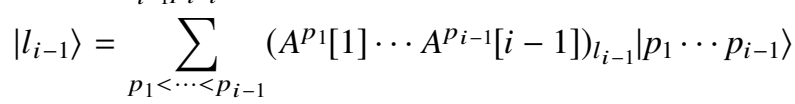

$$
\begin{aligned}
& =\sum_{l_{i-2} p_{i-1}}\left|l_{i-2} p_{i-1}\right\rangle A_{l_{i-2} l_{i-1}}^{p_{i-1}}[i-1],
\end{aligned}
$$

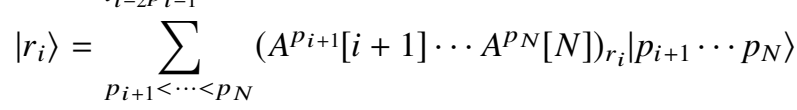

$$
\begin{aligned}
& =\sum_{p_{i+1} r_{i+1}} A_{r_{i} r_{i+1}}^{p_{i+1}}[i+1]\left|p_{i+1} r_{i+1}\right\rangle,
\end{aligned}
$$

where the orbital index $p_{i} \in\{1, \ldots, K\}$ and $\left|p_{1} p_{2} \cdots p_{i}\right\rangle$ are $i$ electron Slater determinants. The summations over $p_{i-1}$ for the states $\left|l_{i-1}\right\rangle$ are purely formal, as the index can take only one value for each $\left|l_{i-1}\right\rangle$, due to the suffix constraint. This also applies to the prefix $p_{i+1}$ for the states $\left|r_{i}\right\rangle$. Thus, the prefix (suffix) of the left (right) renormalized states can be viewed as a kind of "symmetry" index, and a counterpart of the particle number index for renormalized states in Fock space. Both of them represent the $x$-coordinates of the Hilbert subspaces (circles) in the respective configuration graphs, see Figures 1(a) and 1(c). However, unlike the particle number symmetry in Fock space, it should be emphasized that the prefix/suffix "symmetry" is not a physical symmetry of the Hamiltonian, e.g., $\left\langle l_{i}^{\prime}|H| l_{i}\right\rangle \neq 0$ for $\left|l_{i}^{\prime}\right\rangle$ and $\left|l_{i}\right\rangle$ with different suffixes.

From Eq. (8), a HS-MPS representation for $N$-electron wavefunctions can be written down as

$$
|\Psi\rangle=\sum_{p_{1} p_{2} \cdots p_{N}} \Psi^{p_{1} p_{2} \cdots p_{N}}\left|p_{1} p_{2} \cdots p_{K}\right\rangle,
$$

where the tensor $\Psi^{p_{1} p_{2} \cdots p_{N}} \in \mathbb{C}^{N^{K}}$ is given by

$$
\Psi^{p_{1} p_{2} \cdots p_{N}}=\sum_{\left\{\alpha_{i}\right\}} A_{\alpha_{1}}^{p_{1}}[1] A_{\alpha_{1} \alpha_{2}}^{p_{2}}[2] \cdots A_{\alpha_{N-1}}^{p_{N}}[N] .
$$

This is clearly an analogue of FS-MPS (3) for wavefunctions in Fock-space representation (1). The relation of $\Psi^{p_{1}} p_{2} \cdots p_{N}$ (9) with the FCI vector is as follows: Suppose the FCI wavefunction is expressed as

$$
|\Psi\rangle=\sum_{p_{1}<p_{2}<\cdots<p_{N}} \Psi^{\left(p_{1} p_{2} \cdots p_{N}\right)}\left|p_{1} p_{2} \cdots p_{N}\right\rangle,
$$


where $\Psi^{\left(p_{1} p_{2} \cdots p_{N}\right)}$ is the FCI vector with dimension given by the binomial coefficient $C_{K}^{N}$, and $\left(p_{1} p_{2} \cdots p_{N}\right)$ represents an ordered set of orbital indices with $p_{1}<p_{2}<\cdots<p_{N}$. From the way that the HS-MPS is constructed in Figure 1(c), it is easily seen that $\Psi^{p_{1} p_{2} \cdots p_{N}}$ (10) is naturally zero if the orbital indices do not satisfy $p_{1}<p_{2}<\cdots<p_{N}$. Then, FCI wavefunction (9) is obtained through the following relation:

$\Psi^{p_{1} p_{2} \cdots p_{N}}=\left\{\begin{array}{ll}\Psi^{\left(p_{1} p_{2} \cdots p_{N}\right)}, & p_{1}<p_{2}<\cdots<p_{N} \\ 0, & \text { otherwise }\end{array}\right.$.

This gives a "strictly upper triangular" tensor representation of the FCI wavefunction. Such choice differs from the more common antisymmetric tensor representation in Hilbert space, where $\Psi_{A}^{p_{1} p_{2} \cdots p_{N}}=\frac{1}{\sqrt{N !}} \Psi^{\left(p_{1} p_{2} \cdots p_{N}\right)}$ for $p_{1}<p_{2}<\cdots<p_{N}$, and all other entries of the tensor $\Psi^{p_{1} p_{2} \cdots p_{N}}$ are fixed by imposing the antisymmetry, e.g.,

$$
\Psi_{A}^{p_{1} p_{2} \cdots p_{N}}=-\Psi_{A}^{p_{2} p_{1} \cdots p_{N}}=\cdots .
$$

The factor $\frac{1}{\sqrt{N !}}$ arises from the normalization condition. The advantage of the antisymmetric tensor representation is that the set of antisymmetric tensors is closed under the rotation of the single particle basis: It is straightforward to show that the tensor $\tilde{\Psi}^{p_{1} p_{2} \cdots p_{N}}$,

$$
\tilde{\Psi}^{p_{1} p_{2} \cdots p_{N}}=\sum_{p_{1}^{\prime} p_{2}^{\prime} \cdots p_{N}^{\prime}} \Psi_{A}^{p_{1}^{\prime} p_{2}^{\prime} \cdots p_{N}^{\prime}} X_{p_{1}^{\prime} p_{1}} X_{p_{2}^{\prime} p_{2}} \cdots \times X_{p_{N}^{\prime} p_{N}},
$$

which represents the same wavefunction $|\Psi\rangle$ when expressed in another one-particle basis obtained from a transformation $X_{p q}$, is still antisymmetric,

$$
\begin{aligned}
\tilde{\Psi}^{p_{2} p_{1} \cdots p_{N}} & =\sum_{p_{1}^{\prime} p_{2}^{\prime} \cdots p_{N}^{\prime}} \Psi_{A}^{p_{2}^{\prime} p_{1}^{\prime} \cdots p_{N}^{\prime}} X_{p_{2}^{\prime} p_{2}} X_{p_{1}^{\prime} p_{1}} \cdots X_{p_{N}^{\prime} p_{N}} \\
& =\sum_{p_{1}^{\prime} p_{2}^{\prime} \cdots p_{N}^{\prime}}-\Psi_{A}^{p_{1}^{\prime} p_{2}^{\prime} \cdots p_{N}^{\prime}} X_{p_{2}^{\prime} p_{2}} X_{p_{1} p_{1}} \cdots X_{p_{N}^{\prime} p_{N}} \\
& =\sum_{p_{1}^{\prime} p_{2}^{\prime} \cdots p_{N}^{\prime}}-\Psi_{A}^{p_{1}^{\prime} p_{2}^{\prime} \cdots p_{N}^{\prime}} X_{p_{1}^{\prime} p_{1}} X_{p_{2}^{\prime} p_{2}} \cdots X_{p_{N}^{\prime} p_{N}} \\
& =-\tilde{\Psi}^{p_{1} p_{2} \cdots p_{N}},
\end{aligned}
$$

as the multiplications of the numbers $X_{p q}$ commute. It is a particular consequence of the Schur-Weyl duality that relates irreducible finite-dimensional representations of the general linear and symmetric groups, viz., $G L(K)$ and $S_{N}$ in our notation. ${ }^{46}$ Furthermore, if $\Psi_{A}^{p_{1} p_{2} \cdots p_{N}}$ has an MPS representation similar to Eq. (10) obtained by applying successive SVDs, then the MPS representation for the transformed $\tilde{\Psi}^{p_{1} p_{2} \cdots p_{N}}$ can be simply obtained as

$$
\begin{aligned}
\tilde{\Psi}^{p_{1} p_{2} \cdots p_{N}} & =\sum_{\left\{\alpha_{i}\right\}} \tilde{A}_{\alpha_{1}[1]}^{p_{1}}\left[\tilde{A}_{\alpha_{1} \alpha_{2}}^{p_{2}}[2] \cdots \tilde{A}_{\alpha_{N-1}}^{p_{N}}[N],\right. \\
\tilde{A}^{p_{i}}[i] & =\sum_{p_{i}^{\prime}} A^{p_{i}^{\prime}[i] X_{p_{i}^{\prime} p_{i}},}
\end{aligned}
$$

which shows that the bond dimensions are not to be altered by the orbital rotation.

Unfortunately, albeit with these nice formal properties, the antisymmetric tensor representation turns out not to be a good starting point for exploring the Hilbert space MPS representation. Because the antisymmetry generates more nonzero terms in the tensors than "strictly upper-triangular" representation (12), this leads to a significant increase of the bond dimensions (see Sec. IV A for numerical examples). The simplest example to reveal this important defect is to consider the trivial case $(K, N)=(2,2)$, where the dimension of the FCI vector space is one and the wavefunction is simply denoted by $|\Psi\rangle=|12\rangle$. When mapped into the antisymmetric tensor representation, the corresponding tensor (just a matrix in this case) $\Psi_{A}^{p_{1} p_{2}}=\frac{1}{\sqrt{2}}\left[\begin{array}{cc}0 & 1 \\ -1 & 0\end{array}\right]$ is rank-2, which increases the complexity. In comparison, in either the Fock-space representation $\Psi^{n_{1} n_{2}}=\left[\begin{array}{ll}0 & 0 \\ 0 & 1\end{array}\right]$ or Hilbert-space representation (12) $\Psi^{p_{1} p_{2}}=\left[\begin{array}{ll}0 & 1 \\ 0 & 0\end{array}\right]$, the tensors are rank-1. In particular, the latter two representations have the same number of nonzero entries as the original FCI vector. The disadvantage of these two representations is that the closed property of the MPS manifold under orbital rotation no longer holds. For instance, the transformation $X_{p q}$ in Eq. (14) will generally bring nonzero values into the entries of $\Psi^{p_{1} p_{2} \cdots p_{N}}$ violating $p_{1}<p_{2}<\cdots<p_{N}$. In practice, this means a proper ordering of orbitals needs to be chosen. But this seems to be a necessary price to pay when exploring the low-rank structure of fermionic wavefunctions.

\section{B. Particle-hole duality}

Before going into a detailed study of various properties of HS-MPS, we introduce another representation of the wavefunction through using particle-hole duality. Within the finite basis scheme, the same $N$-electron wavefunction can also be understood as a $(K-N)$-hole wavefunction. FCI expansion (11) can be written in terms of determinants of holes $\left|h_{1} h_{2} \cdots h_{K-N}\right\rangle$, which have one-to-one correspondences with determinants of electrons $\left|p_{1} p_{2} \cdots p_{N}\right\rangle$. In CI or FS-MPS, this picture change does not lead to any nontrivial advantage. However, in the case of Hilbert-space MPS it gives a new MPS representation for the same wavefunction, because the sites become $K-N$ holes instead of $N$ electrons. We refer to this new MPS representation as the HS-MPS for holes (HS-MPS[h]) with respect to the fully filled state, and the original representation (10) as theHS-MPS for particles (HS-MPS[p]) with respect to the physical vacuum. In variational calculations, the particle-hole duality implies that rather than minimizing the energy of the second quantized Hamiltonian

$H=\sum_{p q} h_{p q} a_{p}^{\dagger} a_{q}+\frac{1}{2} \sum_{p q r s} g_{p q, r s} a_{p}^{\dagger} a_{q}^{\dagger} a_{s} a_{r} \equiv H_{N}^{p}$,

where $g_{p q, r s}=\langle p q \mid r s\rangle$ in physicists' notation, ${ }^{2}$ using the HS-MPS[p] as an Ansatz, the HS-MPS[h] can alternatively be employed. Here, the notation $H_{N}^{p}$ means the Hamiltonian is normal ordered with respect to the genuine vacuum without electrons. To apply exactly the same variational optimization algorithm to HS-MPS[h], the Hamiltonian $H$ can be simply rewritten in terms of the set of transformed operators $b_{p} \triangleq a_{p}^{\dagger}$ with respect to the fully filled state as

$$
H=\sum_{p q} h_{p q} b_{p} b_{q}^{\dagger}+\frac{1}{2} \sum_{p q r s}\langle p q \mid r s\rangle b_{p} b_{q} b_{s}^{\dagger} b_{r}^{\dagger} .
$$


Through Wick's theorem, ${ }^{4}$ this can be recast into a sum of a constant reference energy for the new vacuum, and a normal ordered Hamiltonian $H_{N}^{h}$ with the same mathematical form as $H_{N}^{p}(17)$,

$$
\begin{aligned}
H & =E_{\mathrm{ref}}+H_{N}^{h}, \\
E_{\mathrm{ref}} & =\sum_{p} h_{p p}+\frac{1}{2} \sum_{p q}\langle p q \| p q\rangle, \\
H_{N}^{h} & =\sum_{p q} \tilde{h}_{p q} b_{p}^{\dagger} b_{q}+\frac{1}{2} \sum_{p q r s} \tilde{g}_{p q, r s} b_{p}^{\dagger} b_{q}^{\dagger} b_{s} b_{r}, \\
\tilde{h}_{p q} & =-h_{q p}-\sum_{s}\langle q s \| p s\rangle, \\
\tilde{g}_{p q, r s} & =g_{p q, r s}^{*}=\langle r s \mid p q\rangle .
\end{aligned}
$$

Unless the bond dimension is sufficiently large to reach the FCI limit, the HS-MPS[p] and HS-MPS[h] with the same maximal bond dimension $D$ generally parametrize different manifolds of states. This point will become clear when examining the theoretical bond dimensions for various CI models in Sec. III C.

\section{Bond dimensions for virtual indices}

In this section, we investigate the minimal bond dimensions $D_{k}$ to represent the FCI or truncated CI spaces by FS/HS-MPS, or in other words, the maximum bond dimensions that are necessary to represent an arbitrary state in a given FCI or truncated CI space. This analysis will be crucial for understanding the performance of FS/HS-MPS.

The starting point is to examine a bipartition of the sites. For simplicity, the Fock space case is considered first. Suppose the sites (orbitals) are (bi)partitioned into two sets and the many-body basis for the left and right Fock spaces are denoted by $\left\{\left|L_{\beta}\right\rangle \triangleq\left|n_{1} \cdots n_{k}\right\rangle\right\}$ and $\left\{\left|R_{\gamma}\right\rangle \triangleq\left|n_{k+1} \cdots n_{K}\right\rangle\right\}$, respectively. Fock space expansion (1) can then be rewritten as

$$
|\Psi\rangle=\sum_{\beta \gamma} \Psi^{L_{\beta} R_{\gamma}}\left|L_{\beta} R_{\gamma}\right\rangle
$$

Meanwhile, the form of FS-MPS (3) allows the wavefunction to be written as a sum of $D_{k}$ terms

$$
\begin{aligned}
&|\Psi\rangle=\sum_{\alpha_{k}}\left|\alpha_{k}^{L} \alpha_{k}^{R}\right\rangle, \\
&\left|\alpha_{k}^{L}\right\rangle \triangleq \sum_{n_{1} \cdots n_{k}}\left(A^{n_{1}}[1] \cdots A^{n_{k}}[k]\right)_{\alpha_{k}}\left|n_{1} \cdots n_{k}\right\rangle, \\
&\left|\alpha_{k}^{R}\right\rangle \triangleq \sum_{n_{k+1} \cdots n_{K}}\left(A^{n_{k+1}}[k+1] \cdots A^{n_{K}}[K]\right)_{\alpha_{k}}\left|n_{k+1} \cdots n_{K}\right\rangle,
\end{aligned}
$$

which is similar to Eq. (7). By comparing Eqs. (20) with (21), one can identify the theoretical bond dimension $D_{k}$ as the rank of the matrix $\Psi^{L_{\beta} R_{\gamma}}$. In particular, if the SVD is applied to $\Psi^{L_{\beta} R_{\gamma}}$, then the resulting decomposition is just the Schmidt decomposition ${ }^{47}$ with the singular values characterizing the entanglement between the two Fock subspaces.

To compute the rank of the matrix $\Psi^{L_{\beta} R_{\gamma}}$, it is also instructive to employ the graphical notation. The dimension of each symmetry sector for $\left\{\left|L_{\beta}\right\rangle\right\}$ can be found in Figure 1(a) or Figure 2(a). To compute the corresponding dimensions for $\left\{\left|R_{\gamma}\right\rangle\right\}$, it is simple to just reverse the flows recursively in

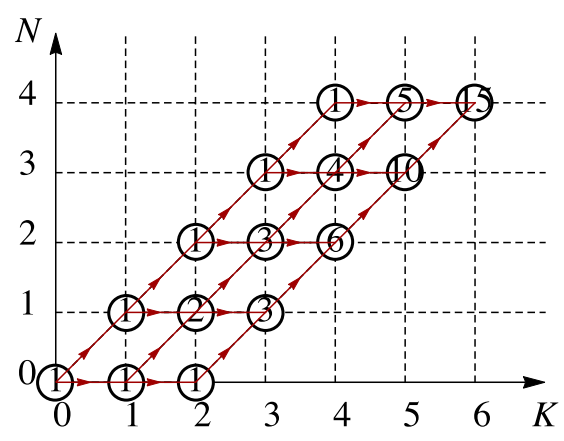

(a)

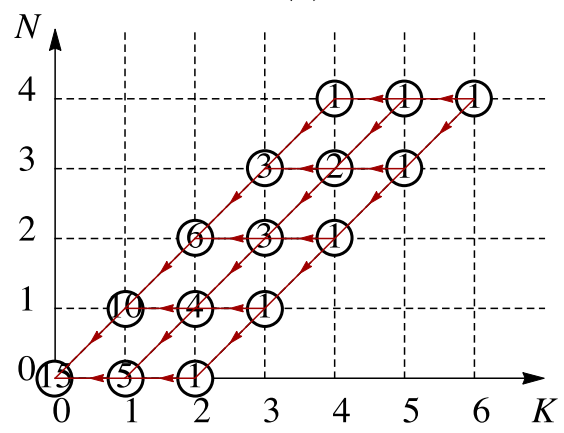

(b)

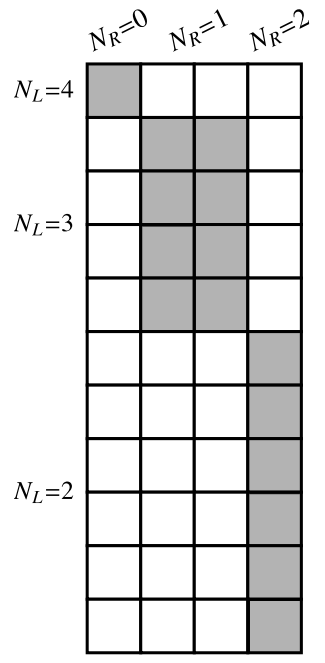

(c)

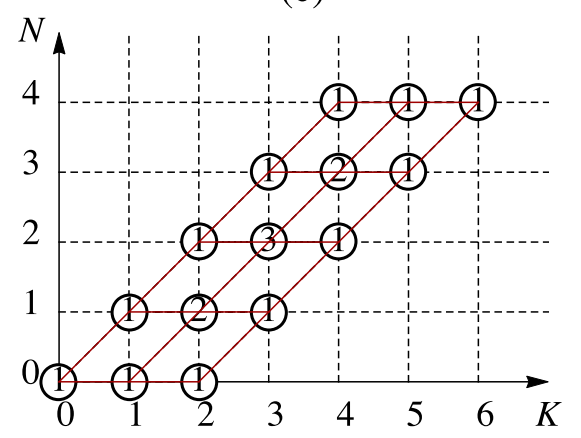

(d)

FIG. 2. Counting the bond dimensions of FS-MPS for $(K, N)=(6,4)$ : (a) dimensions for left Fock spaces $\left\{\left|L_{\beta}\right\rangle \triangleq\left|n_{1} \cdots n_{k}\right\rangle\right\}$, (b) dimensions for right Fock spaces $\left\{\left|R_{\gamma}\right\rangle \triangleq\left|n_{k+1} \cdots n_{K}\right\rangle\right\}$, (c) block structure of $\Psi^{L_{\beta} R_{\gamma}}$ for $k=4$, (d) distributions of bond dimensions in each symmetry sector.

Figure 1(a) starting from the ending point $(K, N)$, which leads to Figure 2(b). Taking $k=4$ as an example, Figure 2(a) shows 
that the left space $\left\{\left|L_{\beta}\right\rangle\right\}$ is composed of three subspaces: $N_{L}=4$ with dimension $1, N_{L}=3$ with dimension 4 , and $N_{L}=2$ with dimension 6 , while Figure 2(b) shows that the complementary right space $\left\{\left|R_{\gamma}\right\rangle\right\}$ is composed of three subspaces: $N_{R}=4-4=0$ with dimension $1, N_{R}=4-3=1$ with dimension 2 , and $N_{R}=4-2=2$ with dimension 1 . Due to the particle number symmetry, $\Psi^{L_{\beta} R_{\gamma}}$ has a block diagonal structure as shown in Figure 2(c), from which the rank can be seen to be the sum of the ranks of smaller blocks. In Figure 2(d), the ranks of smaller blocks computed by simply taking the minimum of the dimensions of left and right subspaces are displayed. The sum of the values on the same layer $k$ gives the theoretical bond dimension $D_{k}$. In the present example, the values of $D_{k}$ are $\{2,4,5,4,2\}$ which can be easily read off from Figure 2(d). It is notable that they are distributed symmetrically about the center of the FS-MPS chain.

The basic principle to compute the bond dimensions for HS-MPS is similar, but there are some differences in the interpretation of the graphs. Figure 3 displays the counterparts of graphs in Figure 2. In the case of HS-MPS, the bipartition of Hilbert space is considered, e.g., $\left\{\left|L_{\beta}\right\rangle \triangleq\left|p_{1} \cdots p_{n}\right\rangle\right\}$ and $\left\{\left|R_{\gamma}\right\rangle \triangleq\left|p_{n+1} \cdots p_{N}\right\rangle\right\}$. Taking $n=3$ as an example, Figure 3(a) shows the dimensions of the subspaces in $\left\{\left|L_{\beta}\right\rangle\right\}$ are 1,3 , and 6 for $\mathcal{P}_{3}^{k=3}, \mathcal{P}_{3}^{k=4}$, and $\mathcal{P}_{3}^{k=5}$, respectively. These are the numbers of configuration strings with the last orbital index equal to a given value $k$. Figure 3(b) shows the reversed process, that is, the configuration strings are grouped by their prefixes, and the values in circles are the numbers of configuration strings whose first orbital index is equal to a given value $k$. Therefore, different from the FS-MPS case, although here the number of rows of $\Psi^{L_{\beta} R_{\gamma}}$ is still given by the numbers in the layer with $n=3$ in Figure 3(a), the number of columns is represented by the numbers in the next layer with $n=4$. This shift of layers for the right space is due to the fact that the reversed process needs to be initiated from the additional fictitious node rather than the node $(K, N)$. Another significant difference between the FS-MPS and HS-MPS cases is that instead of a block diagonal structure for $\Psi^{L_{\beta} R_{\gamma}}$ as in Figure 2(c), the wavefunction $\Psi^{L_{\beta} R_{\gamma}}$ in the Hilbert-space case has a block upper-triangular structure in the bipartitioned basis as shown in Figure 3(c). This difference is again due to the fact that the prefix/suffix indices do not correspond to a physical symmetry; the final wavefunction does not transform as a single "irrep" of the prefix/suffix. When considering renormalization from the left (also simply referred as suffix renormalization), where the recombination of configuration strings is restricted to configuration strings with the same suffix, the bond dimension is given by the sum of ranks for the row-wise blocks with dimensions $(1,3),(3,2)$, and $(6,1)$, which gives $1+2+1=4$ left renormalized states. On the other hand, when considering the renormalization from the right (prefix renormalization), the column-wise blocks are of dimension $(1,1),(1,4)$, and $(1,10)$, which gives only $1+1+1=3$ right renormalized states. Thus, to compute the bond dimensions for suffix renormalization, the dimensions of the row-wise blocks can still be read off from the same layer in Figures 3(a) and 3(b), and their ranks are summarized in Figure 3(d). Again, the sum of the values in the same

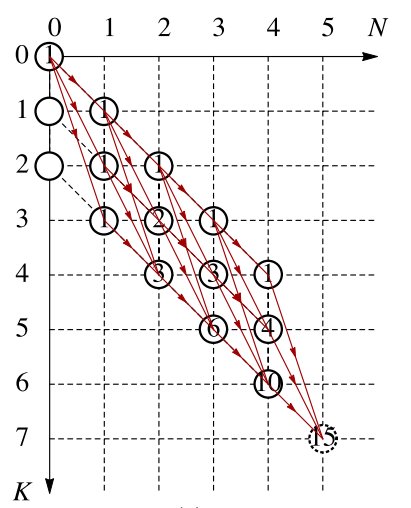

(a)

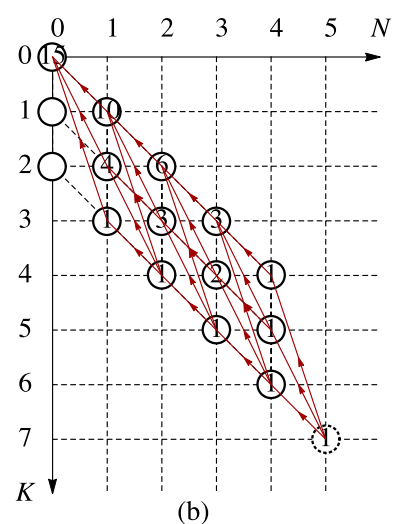

(b)

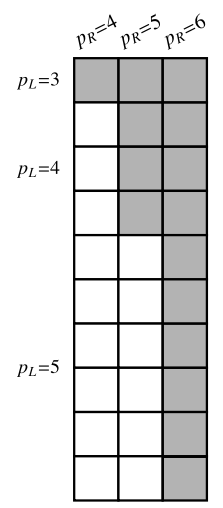

(c)

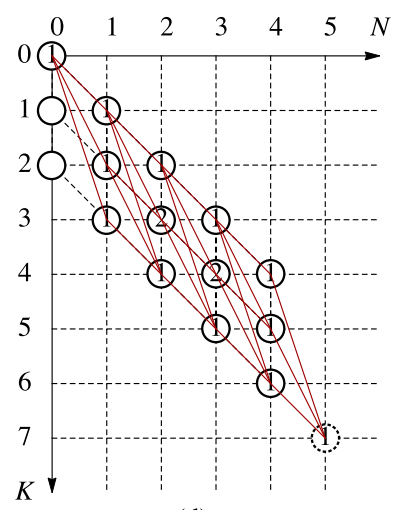

(d)

FIG. 3. Counting the bond dimensions of HS-MPS for $(K, N)=(6,4)$ : (a) dimensions for left Hilbert spaces $\left\{\left|L_{\beta}\right\rangle \triangleq\left|p_{1} \cdots p_{n}\right\rangle\right\}$, (b) dimensions for right Hilbert spaces $\left\{\left|R_{\gamma}\right\rangle \triangleq\left|p_{n+1} \cdots p_{N}\right\rangle\right\}$, (c) block structure of $\Psi^{L_{\beta} R_{\gamma}}$ for $n=3$, (d) distributions of bond dimensions in each symmetry sector.

layer gives a symmetric sequence $\{3,4,4,3\}$. However, there are only three virtual indices for $N=4$, and the additional 
fourth value appears only due to the introduction of the additional node $(K+1, N+1)$. Thus, the fourth value in the sequence does not represent a real bond dimension in the suffix renormalization. This leaves the theoretical bond dimensions $\{3,4,4\}$ for left renormalization in the case $(K, N)=(6,4)$. In contrast, when performing the prefix renormalization from the right, the bond dimensions are given by the sequence $\{4,4,3\}$ by removing the first element in the symmetric sequence. This asymmetric feature is quite different from the FS-MPS case.

The block upper-triangular structure and the structure of renormalization with the prefix/suffix constraints have several important consequences:

First, unlike in the Fock space case where applying successive SVDs to tensor (1) automatically produces an FS-MPS with particle number symmetry, the SVD for Hilbert-space tensor (12) will not lead to a HS-MPS that obeys a prefix/suffix constraint. Rather, it will lead to a more compressed representation because in this case the number of left states is just the rank of $\Psi^{L_{\beta} R_{\gamma}}$, which is three in case of Figure 3(c), rather than 4. However, as we will show in Sec. III G, although combining suffixes can produce a more highly compressed representation, it leads to difficulties in computing matrix elements among the renormalized states. In contrast, matrix elements for the renormalized states with the prefix/suffix constraints can be factorized into products of smaller pieces, which can be computed efficiently in a recursive way similar to the FS-MPS case. This is because the renormalized states with prefix/suffix constraints can be viewed as renormalized states in Fock space, where the prefix/suffix labels the Fock subspace. For instance, a suffix (left) renormalized state $\left|l_{n}\right\rangle$ with a number of electrons $n$ and last orbital index $p$ can also be viewed as a special Fock-space renormalized state $\left|l_{k}\right\rangle$ defined in the Fock subspace $\mathcal{F}_{k=p}$, whose number of electrons is $n$, and where the last orbital $k$ is occupied. In addition, maintaining the constraint leads to theoretical bond dimensions given by sums of ranks of smaller blocks, which is formally similar to the Fock-space case. As will be shown numerically in Sec. IV A, the redundancy introduced by the constraints is in general not large. An interesting example to consider is a set of noninteracting systems. For simplicity, we examine the case where each individual subsystem is an identical closed-shell molecule having $n$ electrons and described by $k$ spin-orbitals. In this case, the exact wavefunction is just a product of wavefunctions of individual systems either in the Fock-space representation or in the Hilbert-space representation without suffix constraints, which means that the bond dimension between the different subsystems is simply one. However, with the prefix/suffix constraint, a bond dimension of $k-n+1$ is needed to fully represent the left renormalized states at the boundary of two subsystems. As long as the maximally allowed bond dimension is larger than this value, then the HS-MPS wavefunction is size extensive, even when there are truncations within each subsystem. For example, as will be shown in Sec. III E, the HS-MPS is flexible enough to represent products of truncated CI wavefunctions. In general, the size extensivity of HS-MPS is ensured by using local orbitals and a proper ordering that groups orbitals belonging to the same subsystem together, which is the same requirement as for size-extensive FS-MPS.

Second, the different bond dimensions for left and right renormalizations implies that when performing onesite DMRG-like optimizations for HS-MPS with a given maximally allowed bond dimension $D$, in general there are truncations during the renormalizations, and this means that the variational energies do not necessarily decay monotonically from site to site during the sweep optimization. However, this is a general feature of MPS whenever a "symmetry" is imposed on the renormalized states, as is the case here with prefix/suffix constraints. For instance, a similar situation happens in the spin-adapted DMRG for non-singlet states. ${ }^{48}$ Consider the following state as an example:

$$
\left.\left.\left.\left.\left|\Psi_{S=1}\right\rangle\right\rangle=\left(\left|l_{S=1}\right\rangle\right\rangle+\left|l_{S=0}\right\rangle\right\rangle\right) \times\left|r_{S=1}\right\rangle\right\rangle
$$

where the double bracket represents the whole spin multiplet including all spin components ${ }^{49}$ and the product $\times$ represents the product with tensor couplings. In a renormalization from the left, $\left.\left|\Psi_{S=1}\right\rangle\right\rangle$ will lead to two spin-adapted left renormalized states $\left(\left|l_{S=1}\right\rangle\right\rangle$ and $\left.\left.\left|l_{S=0}\right\rangle\right\rangle\right)$, whereas it will produce only one spin-adapted right renormalized state $\left.\left|r_{S=1}\right\rangle\right\rangle$ in a renormalization from right. This is analogous to the situation in Figure 3(c).

\section{Exact decomposition of a wavefunction into HS-MPS}

As discussed in Sec. III C, given a wavefunction in tensor representation (12), a direct application of successive SVDs does not lead to Hilbert-space MPS (10). The SVD procedure needs to be modified to incorporate the prefix/suffix constraint. For simplicity, we will only consider the case of decomposing a wavefunction into left canonical form with the suffix constraint. The basic idea to implement the suffix constraint with the SVD is to treat the suffix of the configuration strings as a "symmetry" index as mentioned in Sec. III A. Then, in the direct product of two Hilbert spaces to create a larger Hilbert space, these indices can be used to set up a projection onto the target space whose basis functions obey the ordering $p_{1}<p_{2}<\cdots<p_{N}$. As a simple illustration, we consider the product of the spaces $V_{1}=\{|1\rangle,|2\rangle\}$ and $V_{2}=\{|1\rangle,|2\rangle,|3\rangle\}$ whose "symmetry" indices are just the corresponding orbital indices. The direct product of these two spaces leads to the two-electron configuration space

$$
\begin{aligned}
V_{1} \otimes V_{2} & =\operatorname{span}\{|1\rangle,|2\rangle\} \otimes \operatorname{span}\{|1\rangle,|2\rangle,|3\rangle\} \\
& =\operatorname{span}\{|11\rangle,|12\rangle,|13\rangle,|21\rangle,|22\rangle,|23\rangle\} .
\end{aligned}
$$

Only the basis functions $\{|12\rangle,|13\rangle,|23\rangle\}$ satisfy the requirement $p_{1}<p_{2}$ needed to represent configuration strings for fermions. The first one $|12\rangle$ belongs to the "symmetry" sector with suffix equal to 2 , and the last two $|13\rangle$ and $|23\rangle$ belong to the same "symmetry" sector with suffix equal to 3 . This information can then be reused in the construction of three electron states, if these two-electron states are further coupled with a set of one-electron states $\left\{\left|p_{i}\right\rangle\right\}$. The orbital index "symmetry" can be used during successive SVD procedure (4) to keep track of the combinations that produce configuration strings satisfying $p_{1}<p_{2}<\cdots<p_{N}$ when performing direct 
products of two spaces in chain (5). Once the product of "symmetry" indices is carried out, the SVD can be performed for individual row-wise blocks as in Figure 3(c) to obtain the left renormalized states. Equivalently, such an SVD procedure can also be replaced by the diagonalization of a pseudo-density matrix constructed by taking the diagonal "symmetry" blocks

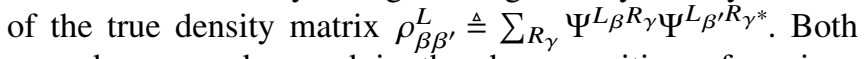
procedures can be used in the decomposition of a given wavefunction into a HS-MPS, or in the decimation step in the DMRG optimization of a HS-MPS, as will be discussed in Sec. III G. For the decimation step, the pseudo-density matrix is slightly more general, as it can be extended to treat multiple states within a state-averaged approach.

\section{E. Restricted manifolds of HS-MPS}

A primary goal of developing the HS-MPS is to use it as a variational Ansatz in the many-electron correlation problem. For instance, the computation of the ground state can be recast into the minimization problem,

$$
E_{0}=\min _{\Psi \in \mathcal{M}} \frac{\langle\Psi|H| \Psi\rangle}{\langle\Psi \mid \Psi\rangle}
$$

where $\mathcal{M}$ represents the manifold of HS-MPS. Although the above discussions have been for the full configuration space, it is rather straightforward to generalize the analysis to truncated configuration spaces. Notably, this can be simply achieved via the graphical representation. In Figure 4, various configuration spaces for the case $(K, N)=(12,6)$ are displayed. These include the Hartree-Fock determinant, CI singles (CIS), doubles, CI singles and doubles (CISD), complete active space with three electrons in six active spin-orbitals $\mathrm{CAS}(3 \mathrm{e}, 6 \mathrm{~s})$, multi-reference CISD (MRCISD) based on this complete active space, doubly occupied CI (DOCI), ${ }^{50}$ and the FCI space. In these various cases, the $\alpha$ and $\beta$ spin orbitals sharing the same spatial part are placed together, and the ordering of the spatial orbitals in the CAS-based methods is doubly occupied, active, and virtual spin orbitals. Note that the order of the orbitals within each category does not change the shapes of the graphs. According to Figure 4, various configuration graphs differ in the accessible nodes and the links among nodes. For instance, the nodes and links for CISD are just the union of those for CIS and doubles, respectively. The DOCI has the same accessible nodes as FCI, but the possible links are restricted such that only the configurations with doubly occupied spatial orbitals are present. When applying the interpretation of renormalization to these graphs, the accessible nodes define the accessible values for the physical indices $p_{i}$ in $A^{p_{i}}[i]$ of HS-MPS. The restrictions on links can be implemented with the help of the orbital index "symmetry" discussed in Sec. III D, which means that in the construction of a tensor product space, the constraints on possible couplings are used in addition to the ordering constraint. In such a way, the HS-MPS can represent all the spaces shown in Figure 4. Moreover, if a fixed bond dimension is used for all the virtual indices as is usually employed in DMRG calculations, then low-rank tensor approximations to these CI models naturally emerge from the HS-MPS. This is true for both HS-MPS[p] and HS-MPS[h]. Note that in principle the FS-MPS can also

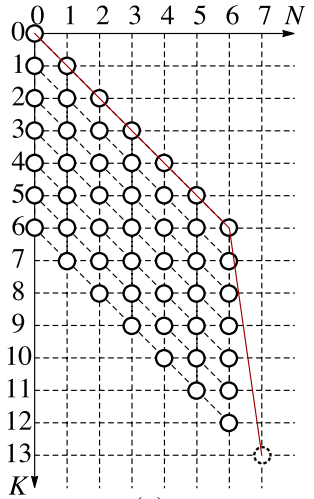

(a)

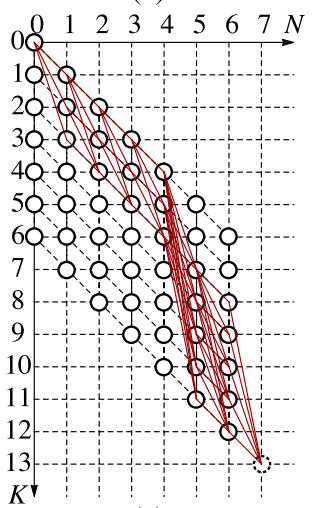

(c)

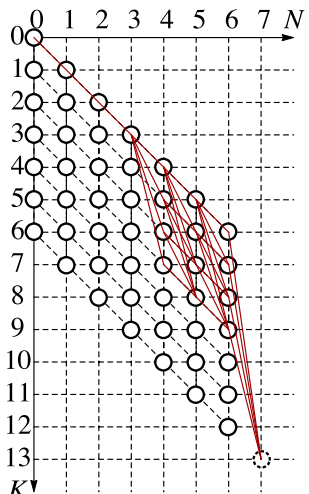

(e)

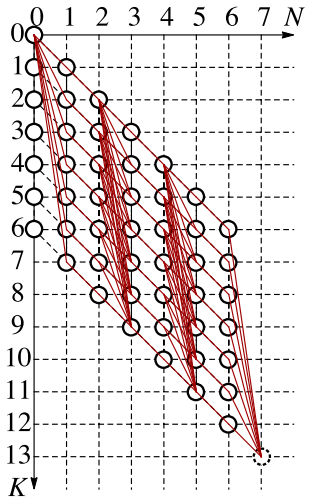

(g)

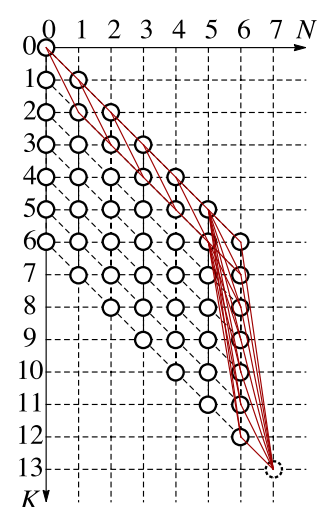

(b)

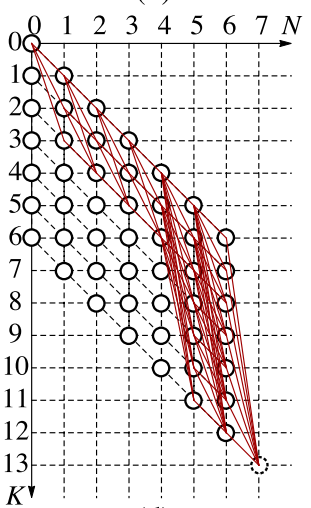

(d)

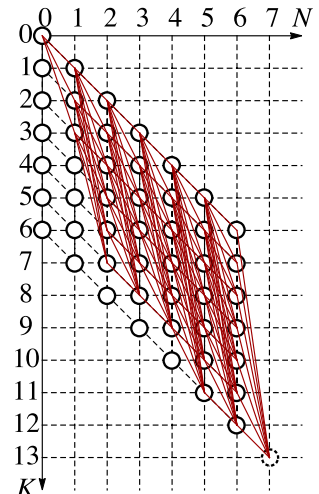

(f)

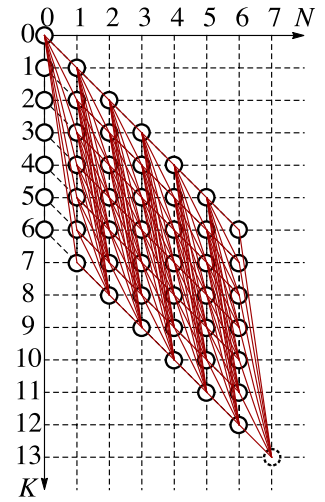

(h)
FIG. 4. Graphical representations of various configuration spaces for $(K, N)=(12,6)$ : (a) Hartree-Fock determinant, (b) CI singles (CIS), (c) doubles, (d) CI singles and doubles (CISD), (e) complete active space (CAS) with three electrons in six active spin-orbitals $\mathrm{CAS}(3 \mathrm{e}, 6 \mathrm{~s})$, (f) multi-reference CISD (MRCISD) based on this complete active space, (g) doubly occupied CI (DOCI), (h) the FCI space.

be used to represent these models, but this requires introducing additional "symmetry" labels that label states by their number 
of particles and holes within the occupied, active, and virtual spaces separately.

The method for counting bond dimensions in Sec. III C also applies to the HS-MPS for truncated CI models. In particular, the comparison between maximal bond dimensions for truncated single-reference CI models helps to better understand the different performances of FS-MPS and HS-MPS. We first consider the half-filled case $(K=2 N)$, where the bond dimensions are the same for HS-MPS[p] and HS-MPS[h]. In Figures 5(a) and 5(b), the distributions of bond dimensions of FS-MPS and HS-MPS for various CI models are illustrated for $(K, N)=(12,6)$, respectively. For the FS-MPS case shown in Figure 5(a), the distributions are all symmetric with the maximum always located in the middle regardless of the excitation level. This is the reason why in DMRG sweeps the minimal energy is usually obtained in the middle,${ }^{51}$ because this corresponds to optimizing the site with the largest number of variational renormalized degrees of freedoms. As shown in Figure 5(b), the distributions for

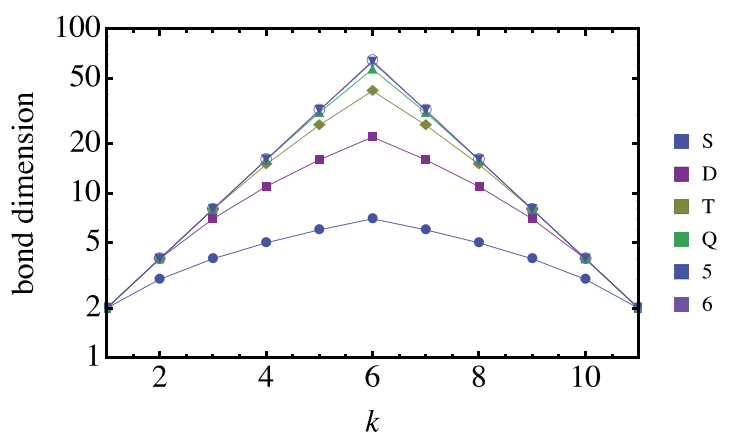

(a)

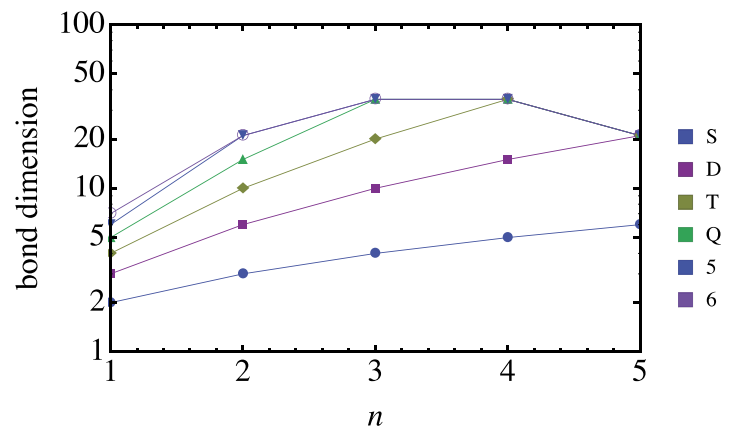

(b)

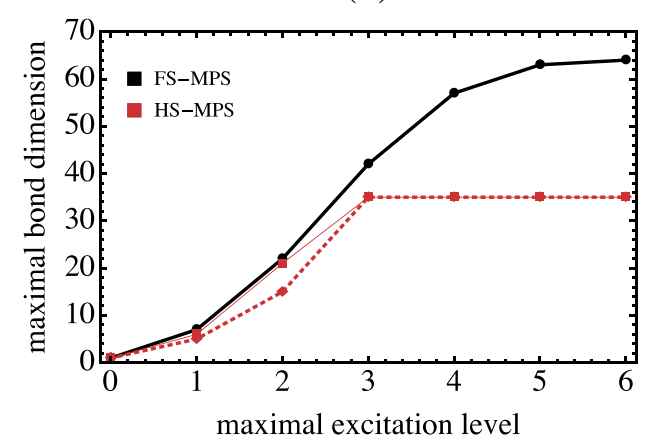

(c)

FIG. 5. Distributions of bond dimensions of FS/HS-MPS for different CI levels in the half-filled case $(K, N)=(12,6)$. (a) $D_{k}$ in FS-MPS. (b) $D_{n}$ in HS-MPS. (c) $D_{\max }$ vs. CI level.
HS-MPS are drastically different, as the maximal bond dimension is located at the right boundary for low excitation levels, and gradually moves to the middle as the maximal excitation level increases. Generally, for the half-filled case the maximal bond dimension of FS-MPS is found to be $2^{N}$, while that of HS-MPS is found to be $2 C_{N}^{(N-1) / 2}$ for odd $N$ and $C_{N+1}^{N / 2}$ for even $N$, respectively. Clearly, all of these scale factorially with $N$.

Figure 5(c) displays the increase of maximal bond dimension versus the increase of the maximal excitation level, which shows that the maximal bond dimension of the HS-MPS is generally smaller than that of the FS-MPS at any excitation level at half-filling. In particular, it becomes saturated after the maximal excitation level exceeds $N / 2$. This phenomenon can be explained by the plot shown in Figure 5(b). As long as the maximal excitation level is greater than $N / 2$, only the bond dimensions for those sites near the left boundary $(n<N / 2)$ are increased. These observations lead to a very practical strategy to maximize the accuracy and computational efficiency of HS-MPS: We can merge the last two sites of HS-MPS into a larger two-site tensor $A^{p_{N-1} p_{N}}[N-1, N]$ and treat it exactly in DMRG sweep optimizations, such that in the CISD case the wavefunctions can be represented exactly with a bond dimension equal to the number of electron pairs $N(N-1) / 2$. This should be compared with the FS-MPS case, where the necessary bond dimension to recover the CISD limit scales as $O(K)$. The effect of this strategy is shown in the red dashed line in Figure 5(c), which shows that the bond dimensions to represent CIS and CISD are reduced, while the computational cost is not greatly increased.

For cases away from half-filling, the HS-MPS[p] and HS-MPS[h] have different structures. Figure 6 shows the distributions of bond dimensions for FS-MPS, HS-MPS[p], and HS-MPS[h] in the case of $(K, N)=(30,10)$. The bond dimensions of FS-MPS for truncated CI models now become less symmetric as shown in Figure 6(a) due to the fact that the configuration graphs for truncated $\mathrm{CI}$ are not symmetric away from the half-filling. In the low-filling limit, the maximal bond dimensions at a given CI level follow the ordering HS-MPS[p] > FS-MPS > HS-MPS[h], suggesting that using the HS-MPS[h] is more effective to represent the CI spaces. For HS-MPS[p], if as mentioned above the last two sites are treated together, then it will be more efficient in recovering the CISD limit (see inset in Figure 6(d)). However, when higher excitations are targeted, HS-MPS[p] becomes less effective. From these two examples with different fillings, we conclude that the relative strengths of the two MPS representations (FS-MPS and HS-MPS) in general depend on the values of $(K, N)$, and also on the actual distribution of configuration coefficients in the CI spaces.

\section{F. Evaluation of matrix elements}

Having defined various manifolds of HS-MPS, now we turn to the evaluations of the matrix elements $\langle\Psi|H| \Psi\rangle$ and $\langle\Psi \mid \Psi\rangle$ in Eq. (24). This is achieved by generalizing the complementary operator approach used in the Fock-space DMRG. ${ }^{16,17,52}$ The simplicity of derivations in FS-MPS case is due to the factorization for the overlap between two 


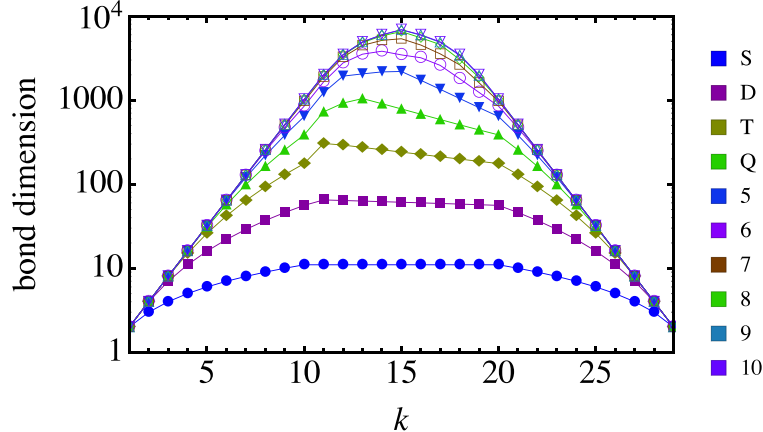

(a)

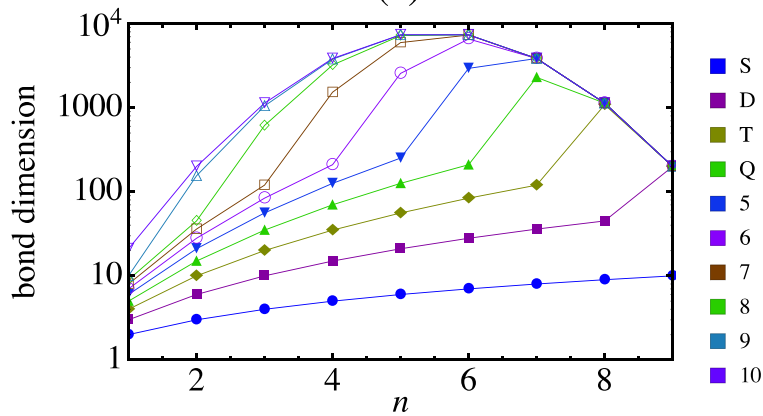

(b)

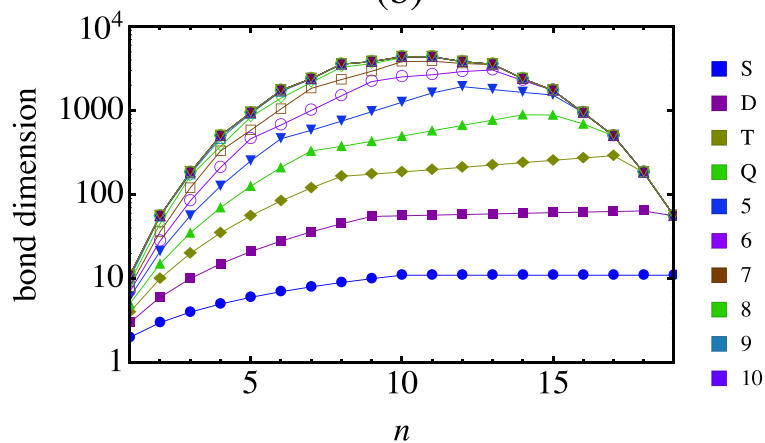

(c)

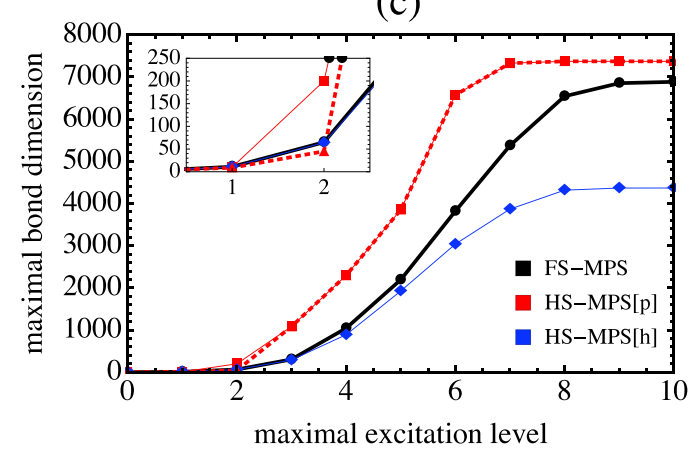

(d)

FIG. 6. Distributions of bond dimensions of FS/HS-MPS for different CI levels in the non-half-filled case $(K, N)=(30,10)$. (a) $D_{k}$ in FS-MPS. (b) $D_{n}$ in HS-MPS[p]. (c) $D_{n}$ in HS-MPS[h]. (d) $D_{\max }$ vs. CI level.

basis vectors

$$
\left\langle n_{1}^{\prime} n_{2}^{\prime} \cdots n_{K}^{\prime} \mid n_{1} n_{2} \cdots n_{K}\right\rangle=\delta_{n_{1}^{\prime} n_{1}} \delta_{n_{2}^{\prime} n_{2}} \cdots \delta_{n_{K}^{\prime} n_{K}} .
$$

By direct application of this fundamental property, it is easy to show that for renormalized states defined by

$$
\left|l_{k}\right\rangle=\sum_{n_{1} \cdots n_{k}}\left|n_{1} \cdots n_{k}\right\rangle L^{n_{1} \cdots n_{k}}
$$

$$
\left|r_{k}\right\rangle=\sum_{n_{k+1} \cdots n_{K}}\left|n_{k+1} \cdots n_{K}\right\rangle R^{n_{k+1} \cdots n_{K}}
$$

the following factorization relation holds for their overlap:

$$
\left\langle l_{k}^{\prime} r_{k}^{\prime} \mid l_{k} r_{k}\right\rangle=\left\langle l_{k}^{\prime} \mid l_{k}\right\rangle\left\langle r_{k}^{\prime} \mid r_{k}\right\rangle,
$$

which breaks the computation of overlaps into products of smaller pieces that can be computed recursively for FS-MPS. In the Hilbert-space case, for two ordered orbital strings $p_{1}^{\prime} p_{2}^{\prime} \cdots p_{N}^{\prime}$ and $p_{1} p_{2} \cdots p_{N}$, a similar factorization still holds

$\left\langle p_{1}^{\prime} p_{2}^{\prime} \cdots p_{N}^{\prime} \mid p_{1} p_{2} \cdots p_{N}\right\rangle=\delta_{p_{1}^{\prime} p_{1}} \delta_{p_{2}^{\prime} p_{2}} \cdots \delta_{p_{N}^{\prime} p_{N}}$.

However, in general for renormalized states defined by

$$
\begin{aligned}
& \left|l_{i}\right\rangle=\sum_{p_{1}<\cdots<p_{i}}\left|p_{1} \cdots p_{i}\right\rangle L^{p_{1} \cdots p_{i}}, \\
& \left|r_{i}\right\rangle=\sum_{p_{i+1}<\cdots<p_{N}}\left|p_{i+1} \cdots p_{N}\right\rangle R^{p_{i+1} \cdots p_{N}},
\end{aligned}
$$

an analogous relation to Eq. (28) does not hold. A counterexample can be simply given as follows:

$$
\begin{aligned}
\left|l_{i}^{\prime} r_{i}^{\prime}\right\rangle & \triangleq(|12\rangle+|56\rangle) \wedge(|34\rangle+|78\rangle), \\
\left|l_{i} r_{i}\right\rangle & \triangleq(|12\rangle+|34\rangle) \wedge(|56\rangle+|78\rangle), \\
\left\langle l_{i}^{\prime} r_{i}^{\prime} \mid l_{i} r_{i}\right\rangle & =\langle 1278 \mid 1278\rangle+\langle 5634 \mid 3456\rangle \\
& \neq\langle 12 \mid 12\rangle\langle 78 \mid 78\rangle=\left\langle l_{i}^{\prime} \mid l_{i}\right\rangle\left\langle r_{i}^{\prime} \mid r_{i}\right\rangle,
\end{aligned}
$$

where $\wedge$ indicates the wedge product of two states in the Hilbert-space case as dictated by the antisymmetry. The violation is due to the exchange of two configuration substrings $|5634\rangle=|3456\rangle$ in $\left|l_{i}^{\prime} r_{i}^{\prime}\right\rangle$; such operations are obviously forbidden in the Fock space by definition. Fortunately, if $\left|l_{i}\right\rangle$ and $\left|r_{i}\right\rangle$ are suffix and prefix renormalized states, respectively, and their combination satisfies the ordering requirements $\left(p_{i}^{\prime}<p_{i+1}^{\prime}\right.$ and $\left.p_{i}<p_{i+1}\right)$, then it can be shown that the simple factorization relation holds

$$
\left\langle l_{i}^{\prime} r_{i}^{\prime} \mid l_{i} r_{i}\right\rangle=\left\langle l_{i}^{\prime} \mid l_{i}\right\rangle\left\langle r_{i}^{\prime} \mid r_{i}\right\rangle,
$$

by directly applying Eq. (29). These conditions simply exclude the situation shown in Eq. (32). The fundamental relation (33) will be extensively used in the following derivation of matrix elements.

To illustrate how the matrix elements of the Hamiltonian can be evaluated in HS-MPS case, the one-electron Hamiltonian is taken as an example. Given the bra state $\left\langle l_{i}^{\prime} r_{i}^{\prime}\right|$ and the ket state $\left|l_{i} r_{i}\right\rangle$, their "symmetry" indices define two partitions for the orbital indices, which can be denoted by $L^{\prime} R^{\prime}$ and $L R$, respectively. The matrix element over $H_{1}$ can thus be separated into a sum of four parts,

$\left\langle l_{i}^{\prime} r_{i}^{\prime}\left|H_{1}\right| l_{i} r_{i}\right\rangle=\sum_{p q \in\left(L^{\prime}+R^{\prime}\right)(L+R)} h_{p q}\left\langle l_{i}^{\prime} r_{i}^{\prime}\left|a_{p}^{\dagger} a_{q}\right| l_{i} r_{i}\right\rangle$.

The summations over $p q \in\left\{L^{\prime} L, R^{\prime} R\right\}$ can be evaluated by using Eq. (33) directly, e.g.,

$$
\begin{aligned}
\sum_{p q \in L^{\prime} L} h_{p q}\left\langle l_{i}^{\prime} r_{i}^{\prime}\left|a_{p}^{\dagger} a_{q}\right| l_{i} r_{i}\right\rangle & =\sum_{p q \in L^{\prime} L} h_{p q}\left\langle\left(a_{p} l_{i}^{\prime}\right) r_{i}^{\prime} \mid\left(a_{q} l_{i}\right) r_{i}\right\rangle \\
& =\sum_{p q \in L^{\prime} L} h_{p q}\left\langle\left(a_{p} l_{i}^{\prime}\right) \mid\left(a_{q} l_{i}\right)\right\rangle\left\langle r_{i}^{\prime} \mid r_{i}\right\rangle \\
& =\left\langle l_{i}^{\prime}\left|H_{1}\right| l_{i}\right\rangle\left\langle r_{i}^{\prime} \mid r_{i}\right\rangle
\end{aligned}
$$


whereas the summations over $p q \in\left\{L^{\prime} R, R^{\prime} L\right\}$ require the expansion of the left and right renormalized states in order to apply Eq. (33), e.g.,

$$
\begin{aligned}
\sum_{p q \in L^{\prime} R} h_{p q}\left\langle l_{i}^{\prime} r_{i}^{\prime}\left|a_{p}^{\dagger} a_{q}\right| l_{i} r_{i}\right\rangle=\sum_{p q \in L^{\prime} R} h_{p q}(-1)^{i}\left\langle\left(a_{p} l_{i}^{\prime}\right) r_{i}^{\prime} \mid l_{i}\left(a_{q} r_{i}\right)\right\rangle \\
=\sum_{p q \in L^{\prime} R} \sum_{p_{i+1}^{\prime} r_{i+1}^{\prime}} \sum_{l_{i-1} p_{i}} h_{p q}(-1)^{i} A_{r_{i}^{\prime} r_{i+1}^{\prime}}^{p_{i+1}^{\prime}}[i+1] \times\left\langle\left(a_{p} l_{i}^{\prime}\right) p_{i+1}^{\prime} r_{i+1}^{\prime} \mid l_{i-1} p_{i}\left(a_{q} r_{i}\right)\right\rangle A_{l_{i-1} l_{i}}^{p_{i}}[i] \\
=\sum_{p q \in L^{\prime} R} \sum_{p_{i+1}^{\prime} r_{i+1}^{\prime}} \sum_{l_{i-1} p_{i}} h_{p q}(-1)^{i} A_{r_{i+1}^{\prime} r_{i+1}^{\prime}}^{p_{\prime}^{\prime} r_{i}^{*}}[i+1] \times\left\langle\left(a_{p} l_{i}^{\prime}\right) \mid l_{i-1}\right\rangle\left\langle p_{i+1}^{\prime} \mid p_{i}\right\rangle\left\langle r_{i+1}^{\prime} \mid\left(a_{q} r_{i}\right)\right\rangle A_{l_{i-1} l_{i}}^{p_{i}}[i] \\
=(-1)^{i} \sum_{p} \sum_{x}\left(\sum_{l_{i-1}}\left\langle l_{i}^{\prime}\left|a_{p}^{\dagger}\right| l_{i-1}\right\rangle A_{l_{i-1} l_{i}}^{x}[i]\right) \times\left(\sum_{r_{i+1}^{\prime}} A_{r_{i}^{\prime} r_{i+1}^{\prime}}^{x *}[i+1]\left\langle r_{i+1}^{\prime}\left|S_{p}\right| r_{i}\right\rangle\right)
\end{aligned}
$$

where the one-electron complementary operator $S_{p}$ is defined by $S_{p} \triangleq \sum_{q} h_{p q} a_{q}$. Note that in both Eqs. (35) and (36), the summation restrictions on $p$ and $q$ have been eliminated in the final expressions, as they are now implicitly imposed by the nonzero conditions of the matrix elements such as $\left\langle l_{i}^{\prime}\left|a_{p}^{\dagger}\right| l_{i-1}\right\rangle$. Thus, Eq. (34) can be finally written as

$$
\begin{aligned}
\left\langle l_{i}^{\prime} r_{i}^{\prime}\left|H_{1}\right| l_{i} r_{i}\right\rangle= & \left\langle l_{i}^{\prime}\left|H_{1}\right| l_{i}\right\rangle\left\langle r_{i}^{\prime} \mid r_{i}\right\rangle+\left\langle l_{i}^{\prime} \mid l_{i}\right\rangle\left\langle r_{i}^{\prime}\left|H_{1}\right| r_{i}\right\rangle+(-1)^{i} \sum_{p} \sum_{x}\left[\left(\sum_{l_{i-1}}\left\langle l_{i}^{\prime}\left|a_{p}^{\dagger}\right| l_{i-1}\right\rangle A_{l_{i-1} l_{i}}^{x}[i]\right)\right. \\
& \left.\times\left(\sum_{r_{i+1}^{\prime}} A_{r_{i}^{\prime} r_{i+1}^{\prime}}^{x *}[i+1]\left\langle r_{i+1}^{\prime}\left|S_{p}\right| r_{i}\right\rangle\right)+\text { c.c. }\right] .
\end{aligned}
$$

In the same way, the expression for total Hamiltonian (17) can be found to be

$$
\begin{aligned}
\left\langle l_{i}^{\prime} r_{i}^{\prime}|H| l_{i} r_{i}\right\rangle= & \left\langle l_{i}^{\prime}|H| l_{i}\right\rangle \times\left\langle r_{i}^{\prime} \mid r_{i}\right\rangle+\left\langle l_{i}^{\prime} \mid l_{i}\right\rangle \times\left\langle r_{i}^{\prime}|H| r_{i}\right\rangle+(-1) \sum_{p r}\left\langle l_{i}^{\prime}\left|a_{p}^{\dagger} a_{r}\right| l_{i}\right\rangle \times\left\langle r_{i}^{\prime}\left|Q_{p r}\right| r_{i}\right\rangle \\
& +\left[(-1)^{i} \sum_{p} \sum_{x}\left\langle l_{i}^{\prime}\left|a_{p}^{\dagger}\right| l_{i-1}\right\rangle A_{l_{i-1} l_{i}}^{x} \times\left\langle A_{r_{i}^{\prime} r_{i+1}^{\prime}}^{x *}\left\langle r_{i+1}^{\prime}\left|\frac{1}{2} S_{p}+S_{p}^{R}\right| r_{i}\right\rangle-\sum_{q}\left(A^{q *} A^{x *}\right)_{r_{i}^{\prime} r_{i+2}^{\prime}}\left\langle r_{i+2}^{\prime}\left|P_{p q}\right| r_{i}\right\rangle\right)\right. \\
& \left.+(-1)^{i} \sum_{q} \sum_{x}\left(A_{l_{i-1}^{\prime}}^{x *} l_{i}^{\prime}\left\langle l_{i-1}^{\prime}\left|\frac{1}{2} S_{q}-S_{q}^{L}\right| l_{i}\right\rangle-(-1)^{i-1} \sum_{p}\left(A^{x *} A^{p *}\right)_{l_{i-2}^{\prime} l_{i}^{\prime}}\left\langle l_{i-2}^{\prime}\left|P_{p q}\right| l_{i}\right\rangle\right) \times\left\langle r_{i}^{\prime}\left|a_{q}^{\dagger}\right| r_{i+1}\right\rangle A_{r_{i} r_{i+1}}^{x}\right) \\
& \left.+\sum_{(p q)} \sum_{x y}\left\langle l_{i}^{\prime}\left|a_{p}^{\dagger} a_{q}^{\dagger}\right| l_{i-2}\right\rangle\left(A^{x} A^{y}\right)_{l_{i-2} l_{i}} \times\left(A^{x *} A^{y *}\right)_{r_{i}^{\prime} r_{i+2}^{\prime}}\left\langle r_{i+2}^{\prime}\left|P_{p q}\right| r_{i}\right\rangle+c . c .\right],
\end{aligned}
$$

where we have omitted the explicit summation notation for $l_{i-1}, r_{i+1}^{\prime}, l_{i-2}$, etc., as well as the site index $k$ in $A[k]$ for brevity, and only the summations over orbital indices are explicitly retained. The additional complementary operators in Eq. (38) are defined by

$$
\begin{aligned}
Q_{p r} & =\sum_{q s} v_{p q r s} a_{q}^{\dagger} a_{s}, \\
P_{p q} & =\sum_{r s} v_{p q r s} a_{r} a_{s}, \\
S_{p}^{R} & =\sum_{q r s} v_{p q r s} a_{q}^{\dagger} a_{r} a_{s}, \\
S_{q}^{L} & =\sum_{p r s} v_{p q r s} a_{p}^{\dagger} a_{r} a_{s},
\end{aligned}
$$

with $v_{p q r s}$ related to the antisymmetrized two-electron integral $\langle p q \| r s\rangle$ by

$$
v_{p q r s}=\left\{\begin{array}{cc}
-\langle p q \| r s\rangle, & p<q, r<s \\
0, & \text { otherwise }
\end{array} .\right.
$$

In summary, Hamiltonian matrix (38) can be factorized into a sum of products just as in the Fock-space case. Albeit with a very complicated form, the physical meaning of Eq. (38) is quite clear: the first two terms are "local" terms, the third term describes density-density (Coulomb and exchange) interaction, and the last three terms describe interactions due to either one-electron or two-electron charge transfers between left and right renormalized states. They all have counterparts in the FS-MPS case. ${ }^{17}$ However, the difference is that here expression (38) explicitly involves the site tensors $A[i-1]$, $A[i], A[i+1]$, and $A[i+2]$, which makes the computation more complicated than the Fock-space case, as discussed in Sec. III G. 


\section{G. Variational optimization by DMRG algorithm}

To perform the optimization in Eq. (24), the DMRG algorithm can be generalized to HS-MPS. The basic idea of DMRG is to optimize the site tensors one-by-one, that is, using a sweep algorithm that optimizes the sites from left to right, and then right to left, until the energy difference between two sweeps converges to below a predefined threshold. For simplicity, we will only discuss the so-called onesite algorithm, and the extension to a two-site algorithm is straightforward. In the one-site algorithm, the tensor $A_{l_{i-1} l_{i}}^{p_{i}}[i]$, or in other words the Hilbert-space counterpart of mapping Eq. (6), is optimized while keeping all other sites fixed. According to (24), this amounts to solving a quadratic optimization problem for $A[i]$ under the left canonical constraint

$$
\sum_{l_{i-1} p_{i}} A_{l_{i-1} l_{i}}^{p_{i^{*}}}[i] A_{l_{i-1} l_{i}^{\prime}}^{p_{i}}[i]=\delta_{l_{i} l_{i}^{\prime}}
$$

Direct gradient-based optimizations are possible for such kind of problems, ${ }^{53}$ but in DMRG this optimization subproblem is solved elegantly via three steps: blocking, solving a CI problem, and decimation.

The central CI part amounts to solving a CI problem in the space spanned by the configurations $\left\{\left|l_{i-1} p_{i} r_{i}\right\rangle\right\}$,

$$
\sum_{l_{i-1} p_{i} r_{i}}\left\langle l_{i-1}^{\prime} p_{i}^{\prime} r_{i}^{\prime}|H| l_{i-1} p_{i} r_{i}\right\rangle \Psi^{l_{i-1} p_{i} r_{i}}=E \Psi l_{i-1}^{\prime} p_{i}^{\prime} r_{i}^{\prime}
$$

where $\left|l_{i-1}\right\rangle$ and $\left|r_{i}\right\rangle$ are the left (suffix) and right (prefix) renormalized states with $i-1$ and $N-i$ electrons, respectively. Graphically, this space is nothing but a contracted CI space as illustrated in Figure 7 for $(K, N)=(6,4)$ and $i=3$, where the colored regions represent the left (red) and right (blue) renormalized states. To solve this problem, in the blocking step the proper superblock space $\left\{\left|L_{i}\right\rangle \triangleq\left|l_{i-1} p_{i}\right\rangle\right\}$ is first formed by combining $\left\{\left|l_{i-1}\right\rangle\right\}$ and $\left\{\left|p_{i}\right\rangle\right\}$ under the ordering constraint by using the orbital index "symmetry." Then standard eigenvalue problem (45) is solved with the Hamiltonian matrix elements computed via Eq. (38). For computational efficiency and memory savings, the matrix representations of operators in the superblock space, e.g., $\left\langle L_{i}^{\prime}\left|a_{p}^{\dagger} a_{r}\right| L_{i}\right\rangle \triangleq\left\langle l_{i-1}^{\prime} p_{i}^{\prime}\left|a_{p}^{\dagger} a_{r}\right| l_{i-1} p_{i}\right\rangle$, are never formed explicitly. They are contracted with the trial vectors denoted by $c_{l_{i-1} p_{i}, r_{i}}$ in the direct CI algorithm, e.g.,

$$
\begin{aligned}
\sigma_{l_{i-1}^{\prime} p_{i}^{\prime}, r_{i}}^{(p r)} \triangleq & \sum_{l_{i-1} p_{i}}\left\langle l_{i-1}^{\prime} p_{i}^{\prime}\left|a_{p}^{\dagger} a_{r}\right| l_{i-1} p_{i}\right\rangle c_{l_{i-1} p_{i}, r_{i}} \\
= & \sum_{l_{i-1}}\left\langle l_{i-1}^{\prime}\left|a_{p}^{\dagger} a_{r}\right| l_{i-1}\right\rangle c_{l_{i-1} p_{i}^{\prime}, r_{i}}-\delta_{p p_{i}^{\prime}} c_{l_{i-1}^{\prime} r}, r_{i} \\
& +(-1)^{i-1}\left[\delta_{p p_{i}^{\prime}} \sum_{l_{i-1} p_{i}}\left\langle l_{i-1}^{\prime}\left|a_{r}\right| l_{i-1} p_{i}\right\rangle c_{l_{i-1} p_{i}, r_{i}}\right. \\
& \left.+\sum_{l_{i-1}}\left\langle l_{i-1}^{\prime} p_{i}^{\prime}\left|a_{p}^{\dagger}\right| l_{i-1}\right\rangle c_{l_{i-1} r, r_{i}}\right] .
\end{aligned}
$$

This reduces the cost from $O\left(K^{4} D^{3}\right)$ in Eq. (46) to $O\left(K^{3} D^{3}\right)$ in Eq. (47), assuming $K \gg N$ and the dimensions of $\left\{\left|l_{i-1}\right\rangle\right\}$ and $\left\{\left|r_{i}\right\rangle\right\}$ are both $D$. This kind of reduction which employs the sparse structure of $\left\langle l_{i-1}^{\prime} p_{i}^{\prime}\left|a_{p}^{\dagger} a_{r}\right| l_{i-1} p_{i}\right\rangle$ is used extensively in the present algorithm, such that the computational cost for the matrix-vector product in the CI problem in the one-site algorithm scales as $O\left(K^{3} D^{3}\right)$. After the wavefunction $\Psi^{l_{i-1} p_{i} r_{i}}$ has been obtained by solving the CI problem, the algorithms in Sec. III D can be employed to obtain $A_{l_{i-1} l_{i}}^{p_{i}}[i]$. In this decimation step, the pseudo-density matrix approach is used, which allows to perform state-average calculations for both ground and excited states and also to add noise to avoid getting stuck in local minimum during the optimizations. With the newly obtained tensor $A_{l_{i-1} l_{i}}^{p_{i}}[i]$, the renormalized operators defined in the contracted space $\left\{\left|l_{i}\right\rangle\right\}$ are also computed directly without constructing the superblock operators. The most time consuming part in this step comes from the renormalization of complementary operators, e.g.,

$$
\begin{aligned}
& \left\langle l_{i}^{\prime}\left|Q_{q s}\right| l_{i}\right\rangle \triangleq \sum_{l_{i-1}^{\prime} p_{i}^{\prime}, l_{i-1} p_{i}} A_{l_{i-1}^{\prime} l_{i}^{\prime}}^{p_{i}^{\prime}{ }^{*}}\left\langle l_{i-1}^{\prime} p_{i}^{\prime}\left|Q_{q s}\right| l_{i} p_{i}\right\rangle A_{l_{i-1} l_{i}}^{p_{i}} \\
& =\sum_{l_{i-1} p_{i}}\left(\sum_{l_{i-1}^{\prime}} A_{l_{i-1}^{\prime} l_{i}^{i^{*}}}^{p_{i}}\left\langle l_{i-1}^{\prime}\left|Q_{q s}\right| l_{i-1}\right\rangle\right) A_{l_{i-1} l_{i}}^{p_{i}} \\
& -\sum_{l_{i-1}^{\prime} p_{i}}\left(\sum_{p_{i}^{\prime}} A_{l_{i-1}^{\prime} l_{i}^{\prime}}^{p_{i}^{\prime}{ }^{\prime}} v_{p_{i}^{\prime} q p_{i} s}\right) A_{l_{i-1}^{\prime} l_{i}}^{p_{i}} \\
& +(-1)^{i-1}\left[\sum_{l_{i-1} p_{i}}\left(\sum_{p}\left\langle l_{i}^{\prime}\left|a_{p}^{\dagger}\right| l_{i-1}\right\rangle v_{p q p_{i}} s\right) A_{l_{i-1} l_{i}}^{p_{i}}\right. \\
& \left.+\sum_{l_{i-1}^{\prime} p_{i}^{\prime}} A_{l_{i-1}^{\prime} l_{i}^{\prime}}^{p_{i}^{\prime *}}\left(\sum_{r} v_{p_{i}^{\prime} q r s}\left\langle l_{i-1}^{\prime}\left|a_{r}\right| l_{i}\right\rangle\right)\right],
\end{aligned}
$$

where the cost has been reduced from $O\left(K^{4} D^{3}+K^{3} D^{3}\right)$ in Eq. (48) to $O\left(K^{3} D^{3}+K^{4} D^{2}\right)$ in Eq. (49).

In summary, since there are $N$ sites that need to be optimized, the computational cost of one sweep in the present one-site algorithm scales as $O\left(N\left(K^{3} D^{3}+K^{4} D^{2}\right)\right)$. In comparison, the corresponding cost for Fock-space DMRG scales $O\left(K^{3} D^{3}+K^{4} D^{2}\right)$, which is cheaper by a factor of $N$. This is mainly due to the fact that the dimension of the physical index in HS-MPS is larger than that in FS-MPS: The former scales as $O(K)$ while the latter is just a constant 2 in spinorbital basis. This makes the two-site algorithm for HS-MPS

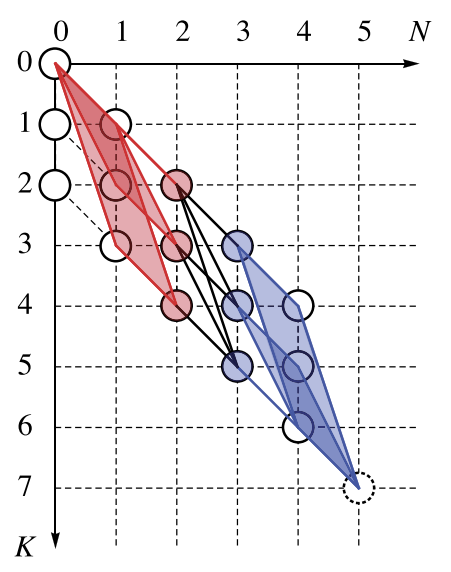

FIG. 7. Contracted configuration space spanned by $\left\{\left|l_{i-1} p_{i} r_{i}\right\rangle\right\}$ for $(K, N)=(6,4)$ and $i=3$. The red circles on the layer $N=2$ represent the subspaces spanned by $\left\{\left|l_{i-1}\right\rangle\right\}$. The blue circle on the layer $N=3$ with $K=p_{i}$ represents the subspace spanned by the direct product of the orbital $p_{i}$ with all $\left\{\left|r_{i}\right\rangle\right\}$. All the orbital index "symmetry"-allowed couplings between $\left\{\left|l_{i-1}\right\rangle\right\}$ and $\left\{\left|p_{i}\right\rangle\right\}$ to form a superblock space $\left\{\left|L_{i}\right\rangle \triangleq\left|l_{i-1} p_{i}\right\rangle\right\}$ are represented by the paths (black lines) between the layers $N=2$ and $N=3$. 
even more expensive, since the dimension of the CI subspace spanned by $\left\{\left|l_{i-1} p_{i} p_{i+1} r_{i+1}\right\rangle\right\}$ becomes $O\left(K^{2} D^{2}\right)$, while in the Fock-space case the corresponding CI dimension is $O\left(D^{2}\right)$ for the space spanned by $\left\{\left|l_{k-1} n_{k} n_{k+1} r_{k+1}\right\rangle\right\}$. Therefore, in the following calculations the one-site algorithm is employed for HS-MPS.

The use of Abelian symmetries ( $S_{z}$ spin projection and $D_{2 h}$ point group symmetry) is rather straightforward. There are two ways to implement $S_{z}$ symmetry. One is to simply separate the $\alpha$ and $\beta$ orbitals into two parts, such that the total wavefunction can be represented by a rectangular matrix $\Psi^{I_{\alpha} J_{\beta}}$, where $I_{\alpha}$ and $J_{\beta}$ are $\alpha$ and $\beta$ strings. Then we can represent both the $\alpha$ and $\beta$ string spaces by HS-MPS, that is, to use two configuration graphs for different spins, and finally connect them by a virtual index $\gamma$, viz., $\Psi^{I_{\alpha} J_{\beta}}=\sum_{\gamma} s_{\gamma} \Psi_{\gamma}^{I_{\alpha}} \Psi_{\gamma}^{J_{\beta}}$ in the SVD form. This works for FCI, but is not quite suitable for the truncated CI models shown in Figure 4. The other more general way to is to use the orbital ordering in Figure 4, and label the renormalized states by a tuple containing the orbital index "symmetry," $S_{z}$ value, and the irreducible representation of the point group, similar to the usage of Abelian symmetries in the FS-MPS case. ${ }^{17}$ The only difference is that the particle number symmetry need not to be considered in HS-MPS, since it has already been taken care of by the Hilbert-space formulation. With this symmetry information, the symmetry-allowed couplings for two spaces in the direct product procedure can be determined, such that the CI space $\left\{\left|l_{i-1} p_{i} r_{i}\right\rangle\right\}$ in the optimization can be symmetryadapted. In the renormalization step, as the pseudo-density matrix is always totally symmetric in the case of Abelian symmetry, it is block diagonal in the superblock space and compatible with the prefix/suffix constraint. Thus, the resulting new renormalized states still carry well defined symmetry properties. The adaptation to non-Abelian symmetry will be discussed in Sec. III H, although we have not implemented in this work.

\section{H. Further generalizations}

Several generalizations of the HS-MPS and the prefix/suffix renormalization can be envisaged.

First, HS-MPS can be extended to represent fully symmetric tensors by representing their independent part, viz., $p_{1} \leq p_{2} \leq \cdots \leq p_{N}$, similar to the antisymmetric case in Eq. (12). This can be used to solve bosonic problems with fixed number of particles. Working with this constraint is as simple as for the fermionic case. Figure 8 displays the corresponding configuration graph for the bosonic case with $(K, N)=(3,4)$, and the HS-MPS is still constructed by adapting the accessible range of physical indices and the coupling rules. In fact, this graph is equivalent to Figure 1(c) or Figure 3(a) for fermions with $(K, N)=(6,4)$ by simply reshaping the graph. In general, there is a one-to-one map between configurations of $N$ fermions in $K$ orbitals and configurations of $N$ bosons in $K^{\prime}=K-N+1$ orbitals, both of which lead to the same space dimension $C_{K}^{N}$. Table I illustrates this mapping for $(K, N)=(6,4)$, where the $i$ th orbital index $p_{i}$ in determinant strings for fermions is mapped to the $i$ th orbital index $p_{i}-i+1$ in permanent strings for

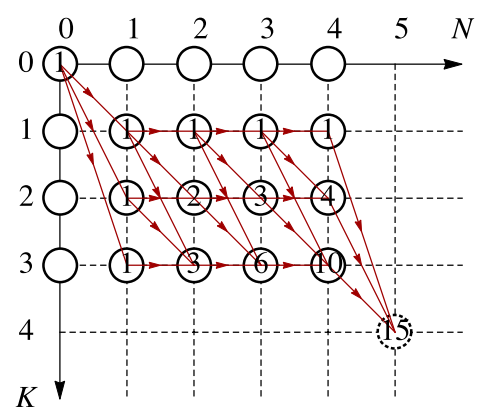

FIG. 8. Configuration graph for bosons with $(K, N)=(3,4)$. The equivalence to Figure 1(c) or Figure 3(a) can be seen simply via reshaping.

bosons ( $i \in\{1, \ldots, N\})$, which is equivalent to the reshaping of the configuration graphs. This correspondence is in fact used in the current implementation for fermions to make the storage of site tensors more compact by employing an effective physical dimension $K^{\prime}=K-N+1$ instead of $K$ for the physical index $p_{i}$.

Next, we propose a sketch of spin adaptation. The advantage of using spin-adapted renormalized states in MPS is the reduction of the bond dimension as exemplified in Sec. IV B as well as the increase of efficiency in the computation of matrix elements by using the Wigner-Eckart theorem. The basic idea of spin adaptation in MPS is to recursively use tensor couplings in the direct product of two spin complete subspaces, which means they are invariant under the action of $S^{2}$ defined in the corresponding underlying Fock spaces, such that the renormalized states can be chosen as eigenfunctions of $S^{2}$. Here, we assume the same spatial orbital is used for both spins, and consequently the number of spin orbitals is even, i.e., $K=2 k$ with $k$ being the number of spatial orbitals. To ensure that every subspace encountered is spin complete, in FS-MPS two adjacent spin-orbital sites are merged into a single site such that the local physical space becomes spin complete, and which can be decomposed into a direct sum of

TABLE I. The correspondences among Fock-space occupation strings for fermions, Slater determinants, and permanents for bosons. The mapping from determinant strings to permanent strings is to change $p_{i}$ to $p_{i}-i+1$ ( $i \in$ $\{1, \ldots, N\}$ ), which corresponds to the reshaping of configuration graphs.

\begin{tabular}{lccc}
\hline \hline No. & Occupation & Determinant & Permanent \\
\hline 1 & 111100 & 1234 & 1111 \\
2 & 111010 & 1235 & 1112 \\
3 & 111001 & 1236 & 1113 \\
4 & 110110 & 1245 & 1122 \\
5 & 110101 & 1246 & 1123 \\
6 & 110011 & 1256 & 1133 \\
7 & 101110 & 1345 & 1222 \\
8 & 101101 & 1346 & 1223 \\
9 & 101011 & 1356 & 1233 \\
10 & 100111 & 1456 & 1333 \\
11 & 011110 & 2345 & 2222 \\
12 & 011101 & 2346 & 2223 \\
13 & 011011 & 2356 & 2233 \\
14 & 010111 & 2456 & 2333 \\
15 & 001111 & 3456 & 3333 \\
\hline \hline
\end{tabular}


two subspaces: $S=0$ with the vacuum and doubly occupied states, and $S=1 / 2$ with singly occupied states. All the intermediate states formed from the tensor coupling of these sites for spatial orbitals can then be made into eigenfunctions of $S^{2}$. This is essentially similar to the construction of a canonical basis, viz., the Gelfand states adapted to the subgroup chain $U(1) \subset U(2) \cdots \subset U(k-1) \subset U(k)$, in the unitary group approach (UGA). ${ }^{43}$

The spin adaptation of HS-MPS is nontrivial, because it is not always natural to obtain spin complete subspaces. For even-electron systems $N=2 n$ as shown in Figure 9(a) for $(K, N)=(6,4)$, the adjacent two sites can be merged into a single site, which forms a spin-complete space for two electrons in $(K-N+2) / 2=k-n+1$ spatial orbitals as

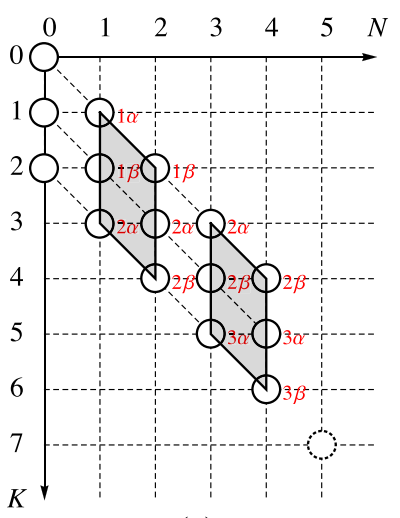

(a)

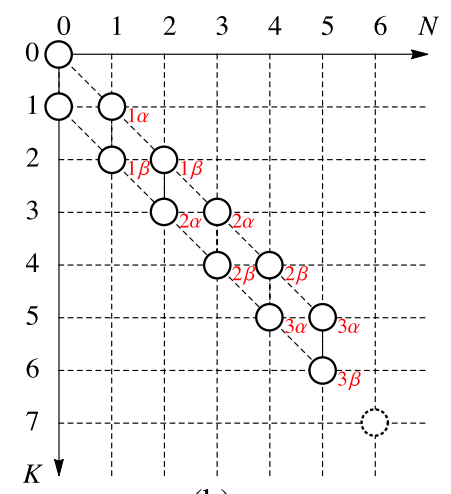

(b)

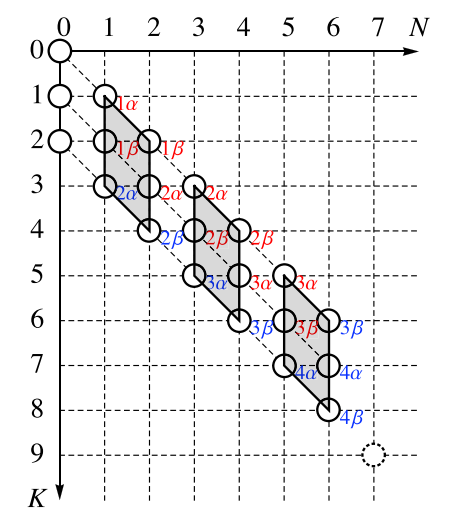

(c)

FIG. 9. On the spin adaptation of HS-MPS for even- and odd-electron systems: (a) $(K, N)=(6,4)$, (b) $(K, N)=(6,5)$, (c) $(K, N)=(6,5)$ combined with a fictitious electron in a fictitious spatial orbital such that $(K, N)=(8,6)$ for the entire system. indicated by the shaded region. The basis for the local physical space corresponding to the new merged physical index can be simply chosen as the set of spin-adapted pairs. Supposing $i<j<k<l$ represent the indices for the spatial orbitals, it is seen that there are two kinds of elementary pairs: the singlet pair formed by one doubly occupied orbital denoted by $\left[i^{2}\right]_{S}$ and the singlet or triplet pairs formed by two singly occupied orbitals denoted by $[i j]_{S, T}$. These pairs can be further coupled to form four-electron states, six-electron states, etc., up to $N=2 n$ electron states. There are five possible ways to couple two electron pairs,

$$
\begin{aligned}
& {\left[i^{2}\right]_{S} \times\left[j^{2}\right]_{S}, \quad\left[i^{2}\right]_{S} \times[j k]_{S, T},} \\
& {[i j]_{S, T} \times\left[k^{2}\right]_{S}, \quad[i j]_{S, T} \times[k l]_{S, T}, \quad[i j]_{S} \times[j k]_{S, T} .}
\end{aligned}
$$

The last one deserves special explanation. Unlike the case $[i j]_{S, T} \times[k l]_{S, T}$, the coupling $[i j]_{S, T} \times[j k]_{S, T}$ can only result in four states as the $j$ th spatial orbital becomes double occupied, viz.,

$$
\begin{aligned}
& {[i j]_{S} \times[j k]_{S}=\left[i j^{2} k\right]_{S},} \\
& {[i j]_{S} \times[j k]_{T}=[i j]_{T} \times[j k]_{S}=\left[i j^{2} k\right]_{T},} \\
& {[i j]_{T} \times[j k]_{T}=\left[i j^{2} k\right]_{S} \oplus\left[i j^{2} k\right]_{T} .}
\end{aligned}
$$

This shows that if all of the couplings are allowed, then there will be a linear dependence among the superblock states in DMRG calculations. Although the resulting CI problem is a generalized eigenvalue problem and can in principle also be solved, for numerical reasons it is usually advantageous to work with MPS without such redundancies. Thus, for simplicity one could just choose the path $[i j]_{S} \times[j k]_{S, T}$ as the only allowed coupling, as in (50). This type of geminal spin-coupling scheme in HS-MPS is in the same spirit as the Serber type spin functions for even number of electrons, ${ }^{54}$ where the spins of two electrons are coupled first and then the spin pairs are coupled sequentially. The DOCI shown in Figure 4 can be viewed as a special case of the spin-adapted HS-MPS, where only the $\left[i^{2}\right]_{S}$ type pairs appear in the construction of the final singlet wavefunction.

The case for odd-electron systems for $(K, N)=(6,5)$ is depicted in Figure 9(b). The same strategy does not work, e.g., the configuration space for the first two electrons spanned by $\left\{\left|1_{\alpha} 1_{\beta}\right\rangle,\left|1_{\alpha} 2_{\alpha}\right\rangle,\left|1_{\beta} 2_{\alpha}\right\rangle\right\}$ is not spin complete due to the lack of $\left|1_{\beta} 2_{\beta}\right\rangle$. On the other hand, combining the first three electrons forms a spin-complete space, but the configuration space for the remaining two does not. To solve this problem, a fictitious electron in a fictitious spatial orbital can be added. This makes the number of electrons even and the pairwise spin adaptation scheme can be applied. For the case shown in Figure 9(b), this approach leads to Figure 9(c), which is quite similar to Figure 9(a). To ensure the correct number of electrons for the physical system, the local space for the last pair must be restricted to the type $[i j]_{S, T}$ where $j$ is the fictitious spatial orbital. Consider the example in Figure 9(c), obtained by applying the coupling scheme in Eq. (50). It is seen that for the first two electrons the only pairs that contribute to the final states are $\left[1^{2}\right]_{S}$ and $[12]_{S}$, the pairs for the next two electrons are $\left[2^{2}\right]_{S}$ and $[23]_{S}$, while the pairs for the last two electron are $[34]_{S, T}$. There are 
only three possible couplings, viz., $\left[1^{2}\right]_{S} \times\left[2^{2}\right]_{S} \times[34]_{S, T}$, $\left[1^{2}\right]_{S} \times[23]_{S} \times[34]_{S, T}$, and $[12]_{S} \times[23]_{S} \times[34]_{S, T}$, such that the dimension of the configuration space is $4 \times 3=12$. It is twice as large as that of the physical system $\left(C_{6}^{5}=6\right)$, as it should be due to the introduction of the fictitious electron and orbital. The use of the fictitious system essentially modifies the structure of the HS-MPS as indicated in Figure 9. Finally, we mention that the idea of using an extra-electron system has been used before e.g., in spin-orbit configuration interaction ${ }^{55}$ to make the Hamiltonian matrix real-symmetric for oddelectron systems.

\section{Connection to other theories}

Before proceeding to numerical studies, we provide several special cases to better understand the HS-MPS Ansatz, and to establish connections with other methods. First, we give an analytic construction of HS-MPS for the simplest nontrivial case $(K, N)=(4,3)$. To this end, FCI wavefunction (11) can be viewed as a multivariate polynomial of orbital indices $p_{i}$, viz.,

$$
f(\mathbf{p})=c_{123} p_{1} p_{2} p_{3}+c_{124} p_{1} p_{2} p_{4}+c_{134} p_{1} p_{3} p_{4}+c_{234} p_{2} p_{3} p_{4},
$$

and the factorization leads to

$$
\begin{aligned}
f(\mathbf{p}) & =p_{1}\left(p_{2}\left(c_{123} p_{3}+c_{124} p_{4}\right)+p_{3}\left(c_{134} p_{4}\right)\right)+p_{2}\left(c_{234} p_{3} p_{4}\right) \\
& =\left[\begin{array}{c}
p_{1} \\
p_{2}
\end{array}\right]^{T}\left[\begin{array}{cc}
p_{2} & c_{124} p_{3} \\
0 & c_{234} p_{3}
\end{array}\right]\left[\begin{array}{c}
c_{123} p_{3}+c_{124} p_{4} \\
p_{4}
\end{array}\right] .
\end{aligned}
$$

From this expression, the nonzero elements of HS-MPS (10) can be read off, and the bond dimensions can be seen to be $\{2,2\}$. Thus, the HS-MPS is related to the factorized form of multivariate polynomials, which are known as multivariate Horner schemes for efficient computations of the value of polynomials. It is a generalization of the well known Horner scheme $^{56}$ for computing the value of a univariate polynomial

$f(x)=\sum_{i=0}^{n} a_{i} x^{i}=a_{0}+x\left(a_{1}+x\left(\cdots\left(a_{n-1}+x a_{n}\right) \cdots\right)\right)$.

Both the HS-MPS and Horner scheme share the same recursive structure.

Next, we examine an interesting class of states that have the HS-MPS as natural representations. We refer to them as "path-weighted" states because their wavefunction coefficients are products of weights for each segment of the path from $(0,0)$ to $(K+1, N+1)$ on the configuration graph for HS-MPS. For example, the nonzero CI coefficient $\Psi^{\left(p_{1} p_{2} p_{3}\right)}$ for $N=3$ can be written as

$$
\Psi^{\left(p_{1} p_{2} p_{3}\right)}=a_{0, p_{1}} b_{p_{1}, p_{2}} c_{p_{2}, p_{3}} d_{p_{3}, K+1}, \quad d_{p_{3}, K+1} \equiv 1,
$$

where $a, b, c, d$ are the weights associated with the path $0 \rightarrow p_{1} \rightarrow p_{2} \rightarrow p_{3} \rightarrow K+1$ for the orbital index on the configuration graph. This kind of state can be represented exactly by a HS-MPS with bond dimension equal to the dimension of the physical index $K-N+1$. Taking $K=5$ as an example, the wavefunction tensor is expressed as

$$
\Psi^{p_{1} p_{2} p_{3}}=A^{p_{1}}[1] A^{p_{2}}[2](A[3] A[4])^{p_{3}} .
$$

In the path-weighted state, the site tensors can then be written as

$$
\begin{aligned}
A[1] & =\left[\begin{array}{l}
a_{0,1} \\
a_{0,2} \\
a_{0,3}
\end{array}\right]^{T}, \quad A[2]=\left[\begin{array}{ccc}
b_{1,2} & b_{1,3} & b_{1,4} \\
0 & b_{2,3} & b_{2,4} \\
0 & 0 & b_{3,4}
\end{array}\right], \\
A[3] & =\left[\begin{array}{ccc}
c_{2,3} & c_{2,4} & c_{2,5} \\
0 & c_{3,4} & c_{3,5} \\
0 & 0 & c_{4,5}
\end{array}\right], \quad A[4]=\left[\begin{array}{l}
1 \\
1 \\
1
\end{array}\right],
\end{aligned}
$$

where the auxiliary site $A[4]$ is introduced just to represent the paths to the virtual site $(K+1, N+1)$. Because of the special structure of the path-weighted state, the tensors for the sites 2 and 3 in Eq. (57) are written in a compact form, and their full (rank-3) forms can be recovered as

$$
A_{\alpha_{1} \alpha_{2}}^{p_{2}}[2]=\left[\left[\begin{array}{ccc}
b_{1,2} & 0 & 0 \\
0 & 0 & 0 \\
0 & 0 & 0
\end{array}\right], \quad\left[\begin{array}{ccc}
0 & b_{1,3} & 0 \\
0 & b_{2,3} & 0 \\
0 & 0 & 0
\end{array}\right], \quad\left[\begin{array}{ccc}
0 & 0 & b_{1,4} \\
0 & 0 & b_{2,4} \\
0 & 0 & b_{3,4}
\end{array}\right]\right] .
$$

The coefficients $b_{p_{i}, p_{j}}$ are a kind of transition amplitude, and left canonical condition (44) ensures that they are normalized as

$$
\begin{aligned}
& \left|b_{1,2}\right|^{2}=1,\left|b_{1,3}\right|^{2}+\left|b_{2,3}\right|^{2}=1, \\
& \left|b_{1,4}\right|^{2}+\left|b_{2,4}\right|^{2}+\left|b_{3,4}\right|^{2}=1 .
\end{aligned}
$$

Furthermore, if the transition amplitudes are site independent and also independent of the source, viz., $(a, b, c)_{i j}=w_{j}$, then the wavefunction can be regarded as a kind of "orbitalweighted" state whose coefficients only depend on the occupied orbitals

$$
\Psi^{\left(p_{1} p_{2} p_{3}\right)}=w_{p_{1}} w_{p_{2}} w_{p_{3}} .
$$

In the extreme case $w_{p}=1$, the state becomes a maximally entangled state,

$$
|\Psi\rangle=\sum_{p_{1}<p_{2}<\cdots<p_{N}}\left|p_{1} p_{2} \cdots p_{N}\right\rangle .
$$

From this analysis, we also observe that there are similarities between both FS-MPS and HS-MPS and artificial neural networks (ANNs). They all share the same recursive 
structure. The virtual indices in MPS can be regarded as the hidden layers in ANNs, the renormalized states can be viewed as neurons, and the site tensors can be seen as the synaptic weights. The physical indices can be interpreted as an additional set of inputs for each layer. Specifically, Figure 2 can be viewed as an ANN with $K-1$ hidden layers, which accepts the vacuum as the input into the origin and outputs the final wavefunction at the site $(K, N)$ via the propagation through the $K-1$ hidden layers by recursive chain (5). The example in Eq. (56) then just corresponds to the single neuron per each orbital index "symmetry" case. The GCF method of Shepard et al. ${ }^{35-39}$ also shares a similar structure, where the contraction is based on the Shavitt $\operatorname{graph}^{43-45}$ for the spin-adapted configuration space. A single $\mathrm{GCF}^{35}$ is in fact a "path-weighted" state, where an arc weight is associated with each path between two nodes in the Shavitt graph. In the latter multifacet generalization, ${ }^{37}$ the number of intermediate states per symmetry sector is allowed to be greater than one. Consequently, the multifacet GCF wavefunction becomes a special spin-adapted Fock-space MPS, where the physical indices are just the step vector in GUGA, the number of renormalized states per symmetry is controlled, and the wavefunction is in either left or right canonical form and optimized by a gradient-based algorithm instead of the DMRG optimization in mixed canonical form. The further connection of spin-adapted FS-MPS with the Shavitt graph will be made clear in Sec. IV B.

We finish with some brief words on entanglement and area laws. A famous property of FS-MPS is that they correctly describe the area law entanglement of 1D ground-states of gapped local Hamiltonians, using a fixed bond dimension $D$. Entanglement is basis dependent, and the above statement applies when using a local site basis, and reflects the locality of the Hamiltonian in that basis, and how it is transferred to its extremal eigenstate. However, entanglement defined in a HS-MPS is not the same as entanglement in a FS-MPS as it involves a partitioning of particles rather than sites. Thus, a direct translation of the area law is difficult. For example, for a single-particle system, there is no entanglement in a HS-MPS at all, while the FS-MPS still describes an entangled state. However, in certain limits where individual particles can be associated with groups of orbitals, such as in the separated atomic limit of a molecular system, then a particle partitioning becomes identical to an orbital partitioning. In that case, the HS-MPS is identical to a kind of FS-MPS where the sites are the groups of orbitals, and the standard area law will apply to the HS-MPS as well.

\section{ILLUSTRATIVE CALCULATIONS}

The DMRG algorithm for HS-MPS has been implemented for generic second quantized Hamiltonians of the form (17). The symmetries of spin projection $S_{z}$ and point groups ( $D_{2 h}$ and its subgroups) have also been implemented. Unless otherwise stated, all the following calculations are carried out using restricted (open-shell) Hartree-Fock orbitals, and transformed molecular integrals are generated by the PyscF package. ${ }^{57}$ The ordering of orbitals in FS-MPS and HS-MPS[p] is chosen in the order of increasing orbital energies, while in HS-MPS[h] such an ordering is reversed to place the orbitals of higher energies in the leftmost sites. The site tensors of the HS-MPS are initialized from the HS-MPS for CISD, and then the physical indices and bond dimensions are gradually increased to the desired values for

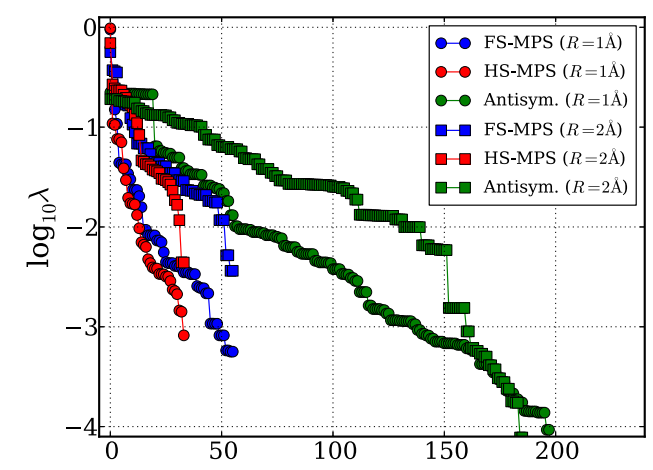

(a)

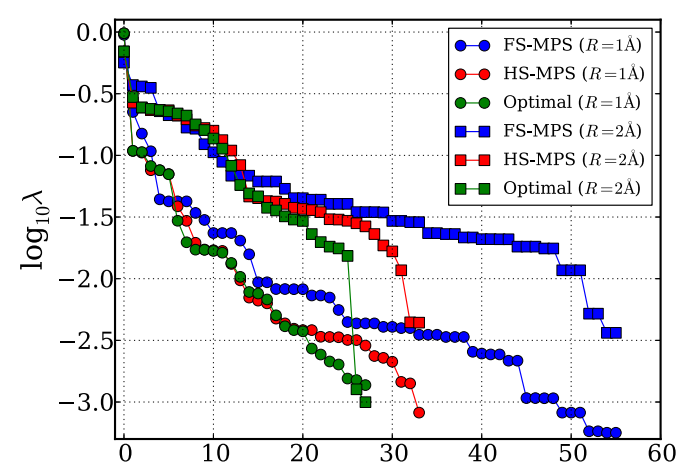

(b)

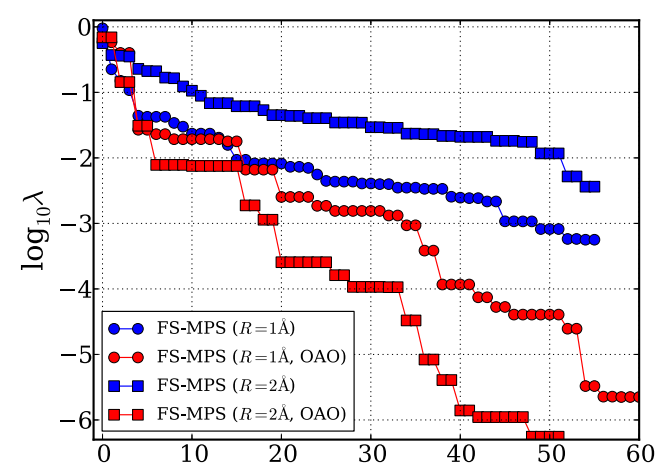

(c)

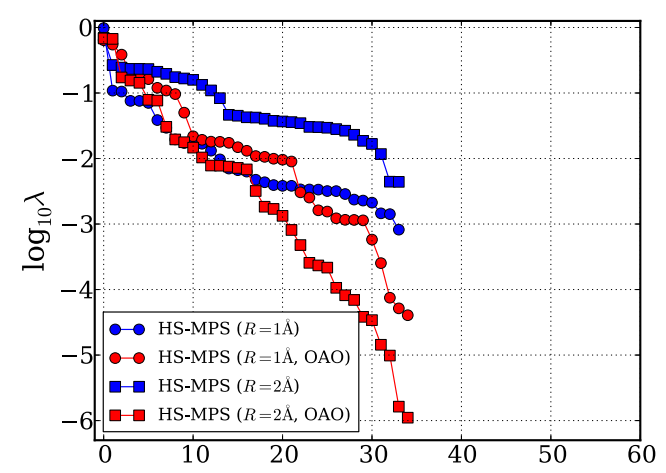

(d)

FIG. 10. The decay of Schmidt values at the middle of various MPS for $\mathrm{H}_{6}$ with $R=1 \AA$ and $R=2 \AA$ 
more accurate calculations. For a fair comparison with the HS-MPS results, the Fock-space DMRG calculations were performed with the BLock code ${ }^{17,18,48}$ using only $S_{z}$ symmetry, unless otherwise stated.

\section{A. Decomposition of many-electron wavefunctions into HS-MPS}

Before discussing the variational calculations, we examine the decomposition of a given FCI wavefunction into HS-MPS using the method presented in Sec. III D. Due to the large memory cost to store the FCI wavefunction tensor, such decompositions cannot been carried out for large $K$ and $N$. The simple linear molecule $\mathrm{H}_{6}$ with bond distance $R=1 \AA$ and $R=2 \AA$ is studied using FCI with the STO-3G basis, which corresponds to the case $(K, N)=(12,6)$ shown in Figure 5. The theoretical bond dimensions for HS-MPS are $\{7,21,35,35,21\}$, while for FS-MPS they are $\{2,4,8,16,32,64,32,16,8,4,2\}$. Figure 10 shows the decay of Schmidt values at the middle of various MPS. As demonstrated in Figure 10(a), even in this simple molecule the singular values decay extremely slowly for the decomposition of antisymmetric Hilbert-space tensor (13). Figure 10(b) shows that the decay rates are comparable for both FS-MPS and HS-MPS. Further, compared with the optimal decomposition without any constraint (see green points in Figure 10(b)), the prefix/suffix constraints in HS-MPS do not introduce too much redundancy. In particular, the rates of decay almost coincide for the first twenty singular values. Clearly, increasing the bond distance makes the system more strongly correlated such that the decay of Schmidt values becomes much more slow. The percentages of configurations with different excitation levels are shown in Figure 11(a), which illustrates the dramatic increase of contributions from higher excitations. The weights of sectors with different orbital index "symmetry" are plotted in Figure 11(a) for $R=1 \AA$ and Figure 11(b) for $R=2 \AA$, respectively. The increase of renormalized states with large populations is clearly revealed when the bond distance is increased.

When localized orbitals are used, the physical picture becomes different. As shown in Figure 10(c), in the Fock space case, the decay of Schmidt values using Löwdin orthonormalized atomic orbitals (OAO) is generally faster than that using canonical orbitals for $R=1 \AA$, while for $R=2 \AA$ the change is more dramatic, viz., the decay is even faster than that for $R=1 \AA$ using OAO. This behavior is well-understood, since the use of localized orbitals minimizes the entanglement. ${ }^{17}$ In particular, the linear hydrogen chain in the large $R$ limit becomes similar to the Heisenberg model with nearest neighbor interactions, such that the area law applies and the bond dimension in FS-MPS can be very small. It is interesting to compare the HS-MPS results shown in Figure 10(d). Indeed, for larger $R$ value, the decay of Schmidt value in terms of OAO is the fastest, which is similar to the FS-MPS case. This is because as discussed above in the large $R$ limit, each electron occupies one spatial orbital such that each physical index in HS-MPS has its own two values corresponding to the two spins, and hence there is no essential difference between HS-MPS and FS-MPS in this

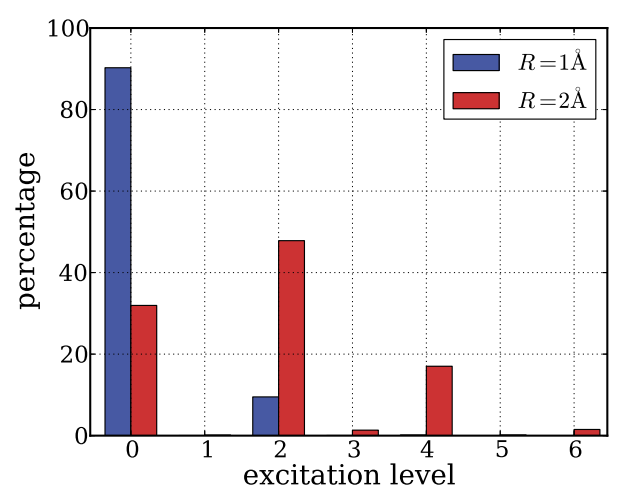

(a)

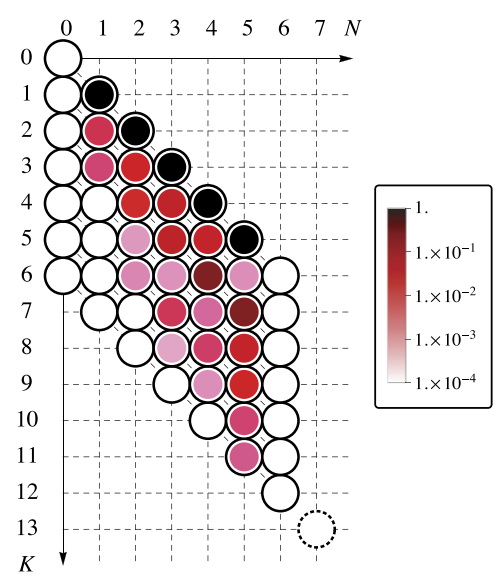

(b)

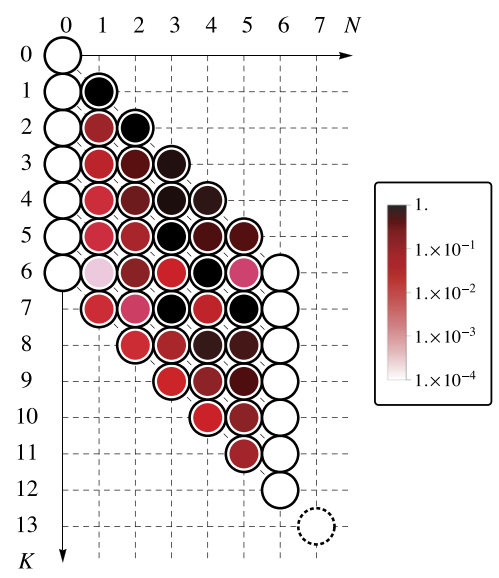

(c)

FIG. 11. Comparison of the FCI wavefunctions and the corresponding HS-MPS for $\mathrm{H}_{6}$ with $R=1 \AA$ and $R=2 \AA$. (a) Population of configurations. (b) Weights of symmetry sectors for $R=1 \AA$. (c) Weights of symmetry sectors for $R=2 \AA$.

limit. However, for $R=1 \AA$ the situation for HS-MPS is more subtle. It is observed that the decay of Schmidt values using canonical orbitals is faster than that using OAO for the first 20 singular values, but becomes slower for the remaining ones. This may suggest that for HS-MPS the canonical orbitals with energetic ordering can have certain advantages over localized orbitals for economically capturing the main features of wave functions, while the remaining part, assumed to describe dynamic correlations, is represented more compactly in terms of local orbitals, due to the local nature of dynamic correlation. 


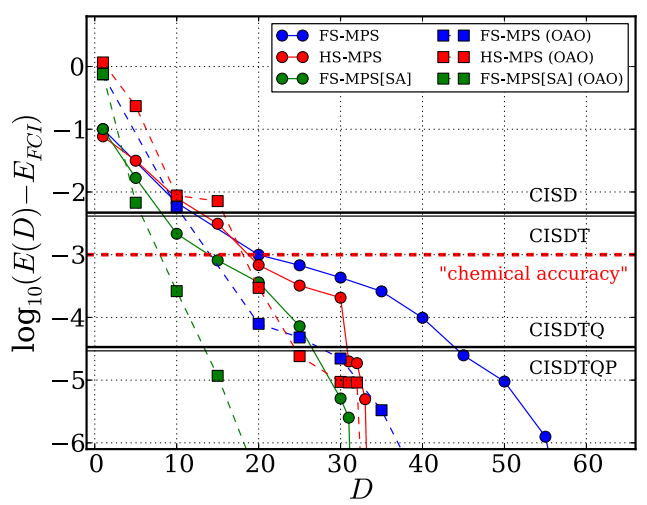

(a)

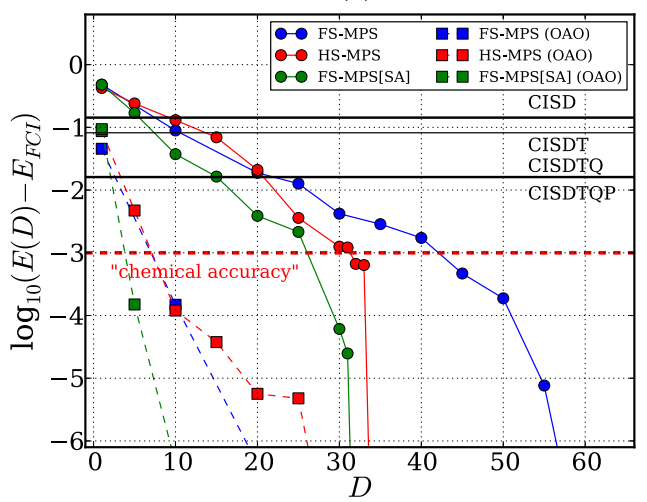

(b)

FIG. 12. Errors of variational energies as a function of $D$ for $\mathrm{H}_{6}$ with $R=1 \AA$ and $R=2 \AA$ in the STO-3G basis. (a) $R=1 \AA$. (b) $R=2 \AA$.

\section{B. Variational calculations of ground states}

Now we turn to the variational calculations via DMRG. Figure 12 shows the errors of variational energies as a function of the maximal bond dimension $D$ for $\mathrm{H}_{6} / \mathrm{STO}-3 \mathrm{G}$ employing FS-MPS and HS-MPS. To illustrate the effect of spin adaptation, the results obtained by spin-adapted FS-MPS are also shown. The red dotted lines represent the so-called "chemical accuracy" $\left(1 m E_{h}\right)$, while the black lines represent the errors of various truncated CI. It is seen that HS-MPS converges faster than FS-MPS for this molecule. This can be traced back to Figure 5(c), which shows that at the half filling the required bond dimension of HS-MPS for a given excitation level is generally smaller than that of FS-MPS. The spin adapted FS-MPS converges even faster, because the spin adaptation reduces the number of renormalized states by using the whole spin multiplet as a single state. This is more evident by plotting the spin-adapted counterpart of the configuration graph, see Figure 13 for the full configuration graph obtained in a similar way as Figure 3(a). The numbers in each block $\left\{\{K, N, S\}, f_{K, N, S}\right\}$ are the number of spatial orbitals $K$, the number of electrons $N$, total spin $S$, and the dimension $f_{K, N, S}$ for this space computed via the Paldus formula. ${ }^{43}$ The physical dimension of spin-adapted FS-MPS is four, indicating four possible couplings between intermediate nodes depending on the changes of particle number $N$ and total spin $S$, viz., $(\Delta N, \Delta S) \in\{(0,0),(1,1 / 2),(1,-1 / 2),(2,0)\}$. Figure 13 is equivalent to the Shavitt graph in GUGA although plotted in a slightly different way, and the four types of couplings correspond to the so-called step vector. ${ }^{44}$ The graph for the right renormalization as a counterpart of Figure 3(b) can be derived by reversing the flows from bottom to top in Figure 13. In this way, the maximal bond dimension can be found at the middle of the hydrogen chain. By summing the dimensions of the subspaces for $K=3$ (blocks in red color), the maximum bond dimension is found to be 35 . This explains the decay pattern of the spin-adapted FS-MPS observed in Figure 12. Finally, it is noteworthy that when using OAO, the FS-MPS converges much faster with respect

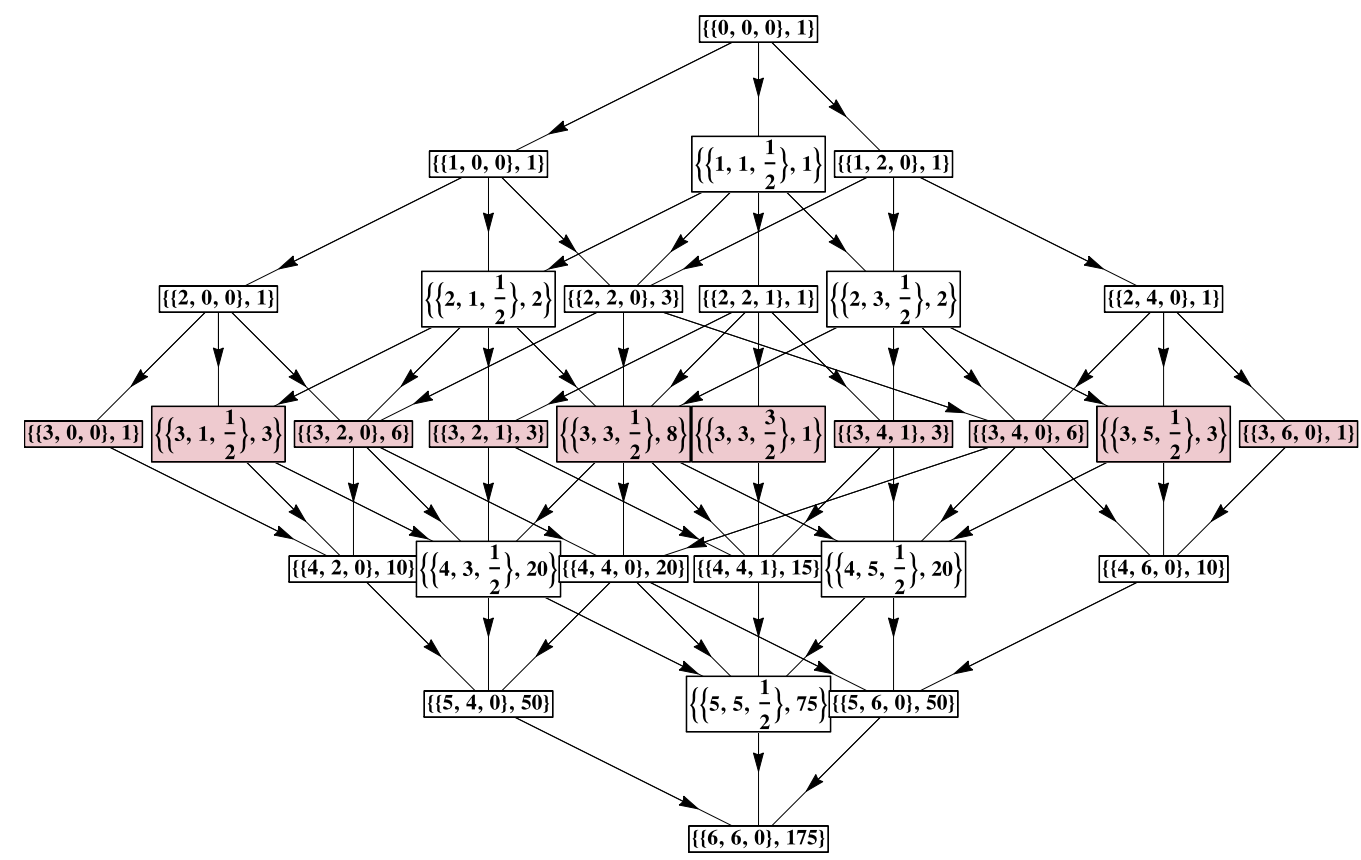

FIG. 13. Configuration graph in terms of spin-adapted intermediate states. It is the same as the Shavitt graph although plotted differently. The numbers in each

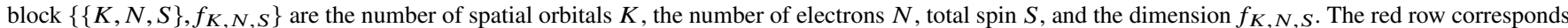
to the largest bond dimension. 
to the increase of $D$ than when using canonical orbitals, in accordance with the decay pattern observed in Figure 10(c). In addition, for $R=1 \AA$ lower energies are obtained by HS-MPS with canonical orbitals for $D$ smaller than ca. 20, while for larger $D$ value HS-MPS with OAO produces lower energies and faster energy convergence with respect to the increase of $D$. This is also in line with the observation for the decay of Schmidt values in Figure 10(d).

For the case away from half filling, we examine the neon atom with Dunning's DZP basis, ${ }^{42,58}$ which corresponds to the case $(K, N)=(30,10)$ shown in Figure 6. In this case, the energies computed by FS-MPS, HS-MPS[p], and HS-MPS[h] at a given bond dimension are generally different as shown in Figure 14. In particular, HS-MPS[h] converges slightly faster than FS-MPS, while HS-MPS[p] converges more slowly than the other two curves. Such behaviors can be anticipated from Figure 6(d), where it is shown that for a given CI level the maximal bond dimension required by HS-MPS[h] is indeed the smallest. More interestingly, according to Figure 14(a), the energies obtained by HS-MPS[p] are lower than those obtained by FS-MPS for $D$ smaller than 100 . This also correlates well with the inset in Figure 6(d), which shows there is a crossing point around 100 between the red dashed line for HS-MPS[p] and the black solid line for FS-MPS. The comparison with various forms of $\mathrm{CI}$ in Figure 14 reveals the important point that to achieve the same accuracy as a given CI model, the bond dimensions required by FS-MPS and HS-MPS with the full set of physical indices are significantly smaller than the

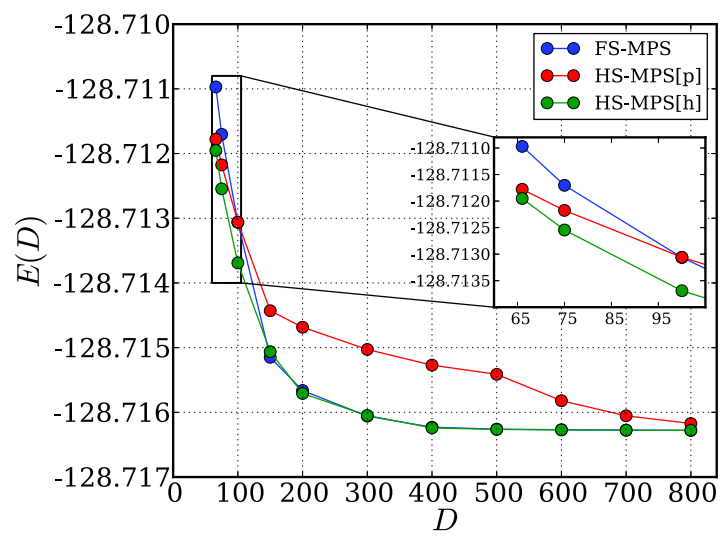

(a)

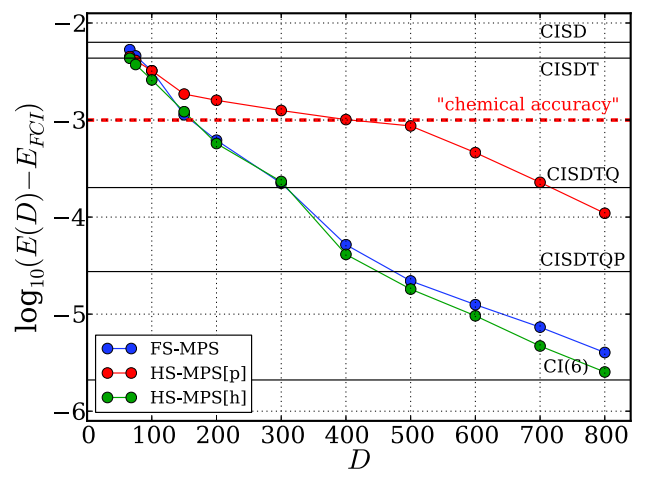

(b)

FIG. 14. Errors of variational energies as a function of $D$ for Ne in the DZP basis. maximal bond dimension shown in Figure 6 for the given maximal CI level. For instance, to achieve CISDTQ accuracy, the bond dimensions required by FS-MPS, HS-MPS[p], and HS-MPS[h] are about 300, 700, and 300, respectively. All of these are much smaller than the maximal bond dimensions shown in Figure 6, which are 1044, 2295, and 890 for FS-MPS, HS-MPS[p], and HS-MPS[h], respectively. The fundamental reason is that the configurations included in a truncated CI model based on excitation rank do not contribute equally to the correlation energies. Further, some of the excluded higher excitations in a truncated CI model may be more important. This is particularly true in the strong correlation regime, as will be shown in the next example, where it can be seen that the FS/HS-MPS samples the full set of important configurations very efficiently.

The water molecule $\mathrm{H}_{2} \mathrm{O}$ with geometry $\left(R_{\mathrm{OH}}=1 \AA\right)$ taken from Ref. 59 is employed as an example to test the convergence of HS-MPS[p] for various truncated CI models. Table II shows the results computed with Dunning's DZ basis, ${ }^{58}$ which corresponds to $(K, N)=(28,10)$. The active spaces for CASCI and MRCISD were 6-electron-in-8-spatialorbital CAS $(6 \mathrm{e}, 8 \mathrm{o})$ with Hartree-Fock orbitals. It is seen that both CISDTQ and MRCISD reach $1 m E_{h}$ accuracy in this case, although they cover only a small portion of the FCI space. The convergence of HS-MPS for these two models is similar to the convergence for FCI, and the $1 m E_{h}$ accuracy is reached at around $D=300$ in all cases (CISDTQ, MRCISD, and FCI). It is also interesting to compare the results of HS-MPS $(D=45)$ with the CISD results. The CISD wave function can be exactly represented by a HS-MPS with $D=45$ and a restricted set of physical indices, see the first column of Table II. Then, it is apparently surprising to see that with the same bond dimension $D=45$, an increase of error $\left(21.2 m E_{h}\right)$ is observed when the

TABLE II. Convergence of HS-MPS for various CI models (energy in $m E_{h}$ ) for $\mathrm{H}_{2} \mathrm{O}$ with the $\mathrm{DZ}$ basis.

\begin{tabular}{lcccccc}
\hline \hline$D$ & CISD & CISDT & CISDTQ & CASCI & MRCISD & FCI \\
\hline \multicolumn{5}{c}{$R_{\mathrm{OH}}=1 \AA$ A,$E_{F C I}=-76.156699 E_{h}$} \\
Error $^{\mathrm{a}}$ & 8.3 & 7.1 & 0.3 & 90.2 & 0.8 & 0.0 \\
\hline 45 & 0.0 & 15.8 & 21.0 & 0.4 & 30.0 & 21.2 \\
100 & 0.0 & 0.5 & 4.0 & 0.0 & 7.6 & 4.2 \\
150 & 0.0 & 0.0 & 2.3 & 0.0 & 1.8 & 2.4 \\
200 & 0.0 & 0.0 & 1.4 & 0.0 & 1.2 & 1.5 \\
300 & 0.0 & 0.0 & 1.0 & 0.0 & 0.6 & 1.1 \\
400 & 0.0 & 0.0 & 0.4 & 0.0 & 0.4 & 0.5 \\
500 & 0.0 & 0.0 & 0.1 & 0.0 & 0.2 & 0.3 \\
\hline & \multicolumn{7}{c}{$R_{\mathrm{OH}}=3 \AA, E_{F C I}=-75.866$} & $193 E_{h}$ & \\
Error & 131.9 & 100.5 & 12.3 & 63.0 & 0.5 & 0.0 \\
\hline 45 & 0.0 & 12.8 & 34.6 & 8.4 & 57.7 & $42.8,46.9^{\mathrm{b}}$ \\
100 & 0.0 & 1.6 & 11.8 & 0.3 & 38.9 & $17.6,20.7^{\mathrm{b}}$ \\
150 & 0.0 & 0.0 & 2.7 & 0.0 & 15.3 & $5.8,5.9^{\mathrm{b}}$ \\
200 & 0.0 & 0.0 & 2.0 & 0.0 & 5.6 & $3.9,2.8^{\mathrm{b}}$ \\
300 & 0.0 & 0.0 & 1.0 & 0.0 & 1.5 & $1.9,1.6^{\mathrm{b}}$ \\
400 & 0.0 & 0.0 & 0.8 & 0.0 & 1.1 & $1.2,0.8^{\mathrm{b}}$ \\
500 & 0.0 & 0.0 & 0.2 & 0.0 & 1.0 & $1.1,0.5^{\mathrm{b}}$ \\
\hline \hline
\end{tabular}

${ }^{\mathrm{a}}$ Errors of truncated CI relative to FCI energies.

${ }^{\mathrm{b}}$ State-average DMRG results. 
physical indices are not restricted in the HS-MPS for FCI, as compared with the CISD error $\left(8.3 m E_{h}\right)$. This, however, is because if there are truncations in the DMRG, which is the case for HS-MPS when using the one-site algorithm (although not the case for FS-MPS) then the DMRG algorithm does not strictly correspond to an energy minimization. In particular, the density matrix criterion for choosing the left and right renormalized space is no longer optimal with respect to the energy metric. However, when the bond dimension becomes larger, the error will be reduced again, e.g., the error becomes $4.2 m E_{h}$ when $D$ goes to 100 , which is better than the CISD energy.

It is also interesting to examine a case of static correlation, that arises when the water molecule is symmetrically stretched to $R_{\mathrm{OH}}=3 \AA$. To avoid convergence to the wrong spin state, we used a "biased" Hamiltonian with $\lambda=1$, viz.,

$$
H(\lambda)=H+V(\lambda), \quad V(\lambda)=\lambda S_{-} S_{+},
$$

to shift the energies of states with spin $S>0$ upwards. Alternatively, we also performed state average calculations targeting two states at a time without adding the bias, in the case of FCI. This approach leads to slightly faster convergence as shown in Table II. It is notable that while CISDTQ itself has a large error, of about $12 m E_{h}$, the HS-MPS for the various $\mathrm{CI}$ models displays similar convergence patterns as for the case of $R_{\mathrm{OH}}=1 \AA$, and thus the error of HS-MPS is quite "parallel" to the parent model. The data further show that the HS-MPS for FCI captures many important higher excitations at a bond dimension of around 400, due to the unrestricted physical dimension, as compared with HS-MPS for CISDTQ with the same bond dimension where the physical dimensions are restricted.

\section{State-average calculations of excited states}

While it is seen for the $K>N$ case that the FS-MPS generally provides better energies as compared with HS-MPS[p], we can expect HS-MPS[p] to be more efficient for describing a manifold of low-lying states. There are several reasons to support this. First, one major difference between HS-MPS[p] and FS-MPS is that HS-MPS[p] has a larger physical dimension $K-N+1$ compared to 2 in the FS-MPS. This results in a much larger $\mathrm{CI}$ subproblem during the DMRG site optimization, such that the excited states can be expected to be better described in the local variational space of a given site. Second, considering the extreme case where all lowlying states arise from excitations from the highest occupied molecular orbital (HOMO) to the virtual orbitals, to represent all such states in a HS-MPS[p] only the last site needs to be changed. As in our setting the last two sites of HS-MPS[p] are treated exactly without any truncation, this implies that all these excited states can be described without increasing the bond dimension because they can share the same $(N-1)$ electron states. In the occupation number representation used in the FS-MPS, to represent all these excitations the bond dimension will at least increase by one over that for a single state.

To test this expectation, state-averaged calculations were performed for the lowest six states of $\mathrm{H}_{2} \mathrm{O}$ with the same basis
TABLE III. Errors with respect to FCI energies (in $m E_{h}$ ) for the lowest six states of $\mathrm{H}_{2} \mathrm{O}$ in the DZ basis.

\begin{tabular}{|c|c|c|c|c|c|c|c|c|c|c|}
\hline \multirow[b]{2}{*}{ State } & \multirow[b]{2}{*}{$\mathrm{FCI} / E_{h}$} & \multirow[b]{2}{*}{ CISD } & \multicolumn{4}{|c|}{ FS-MPS $(D)$} & \multicolumn{4}{|c|}{ HS-MPS $(D)$} \\
\hline & & & 45 & 100 & 200 & 400 & 45 & 100 & 200 & 400 \\
\hline $1^{1} A_{1}$ & -76.156699 & 8.3 & 40.3 & 20.0 & 4.9 & 1.5 & 29.9 & 20.6 & 3.2 & 1.3 \\
\hline $1^{3} B_{2}$ & -75.876664 & 85.9 & 49.3 & 24.6 & 5.2 & 1.8 & 46.9 & 22.3 & 3.9 & 2.7 \\
\hline $1^{1} B_{2}$ & -75.847464 & 85.5 & 48.0 & 24.5 & 5.1 & 1.7 & 45.3 & 22.3 & 4.1 & 2.6 \\
\hline $1^{3} A_{1}$ & -75.798565 & 85.0 & 65.2 & 25.3 & 4.9 & 1.9 & 55.5 & 23.9 & 4.8 & 3.2 \\
\hline $1^{3} A_{2}$ & -75.793979 & 86.3 & 62.0 & 28.0 & 9.6 & 2.1 & 54.6 & 22.6 & 4.7 & 2.7 \\
\hline $1^{1} A_{2}$ & -75.774742 & 84.9 & 60.4 & 28.3 & 10.5 & 2.1 & 53.6 & 22.3 & 4.8 & 2.6 \\
\hline MAE & & 72.6 & 54.2 & 25.1 & 6.7 & 1.8 & 47.7 & 22.3 & 4.2 & 2.5 \\
\hline $1^{1} A_{1}{ }^{\mathrm{a}}$ & -76.156699 & 8.3 & 8.0 & 3.5 & 1.1 & 0.1 & 21.2 & 4.2 & 1.6 & 0.5 \\
\hline
\end{tabular}

${ }^{\mathrm{a}}$ Single-state DMRG results.

set and geometries $\left(R_{\mathrm{OH}}=1 \AA\right)$ as employed in Sec. IV B. Only the $S_{z}=0$ sector is targeted and states with different spatial symmetries are all state-averaged in the construction of the pseudo-density matrix for renormalizations. Table III lists the computed energies with FS/HS-MPS, and for comparison the results from single-state optimizations are also shown. The most striking feature is that when going from single-state to multi-state optimizations, the mean absolute errors (MAEs) of the FS-MPS with relatively small bond dimensions increase significantly by a factor about 6-7. In comparison, the MAEs of HS-MPS do not increase dramatically. Consequently, increasing the number of states makes the MAEs of the HS-MPS become smaller than those of the FS-MPS with bond dimensions 45,100 , and 200 . When the bond dimension is sufficiently large (around 400), then again the MAEs of the FS-MPS become smaller than those of the HS-MPS as in the single-state calculations. The comparison with the CISD results is also interesting. The CISD results indicate a large bias towards the ground state as the orbitals are optimized for the ground state. However, as shown in Table III, HS-MPS with $D=45$ already rectifies this bias by adjusting the structure of the renormalized states. Consequently, the MAE of HS-MPS with $D=45$ becomes much smaller than that of CISD in the multi-state calculations.

TABLE IV. Errors with respect to FCI energies (in $m E_{h}$ ) for the lowest five $\Sigma^{+}$states and four $\Pi$ states of $\mathrm{BeH}$ in the aug-cc-pVDZ basis.

\begin{tabular}{|c|c|c|c|c|c|c|c|c|c|c|}
\hline \multirow[b]{2}{*}{ State } & \multirow[b]{2}{*}{$\mathrm{FCI} / E_{h}$} & \multirow[b]{2}{*}{ CISD } & \multicolumn{4}{|c|}{ FS-MPS $(D)$} & \multicolumn{4}{|c|}{ HS-MPS ( $D)$} \\
\hline & & & 10 & 50 & 100 & 200 & 10 & 50 & 100 & 200 \\
\hline${ }^{2} \Sigma^{+}$ & -15 & 0.8 & 23.6 & 2.4 & 1.3 & 0.1 & 1.1 & 0.6 & 0.2 & 0.1 \\
\hline${ }^{2} \Sigma^{+}$ & -14.9 & 12.8 & 28.6 & 3.5 & 1.8 & 0.1 & 6.0 & 1.7 & 0.4 & 0.2 \\
\hline $3^{2} \Sigma^{+}$ & -14.98 & 27.2 & 35.4 & 5.9 & 3.9 & 0.1 & 9.9 & 3.8 & 0.7 & 0.3 \\
\hline $4^{2} \Sigma^{+}$ & -14 & 28.2 & 34.6 & 2.6 & 1.4 & 0.1 & 8.6 & 1.8 & 0.2 & 0.1 \\
\hline $5^{2} \Sigma^{+}$ & -14 & 25.5 & 41.8 & 6.2 & 4.9 & 0.1 & 24.7 & 4.7 & 0.6 & 0.2 \\
\hline MAE & & 18.9 & 32.8 & 4.1 & 2.7 & 0.1 & 10.1 & 2.5 & 0.4 & 0.2 \\
\hline${ }^{2} \Pi$ & -10.098914 & 26.3 & 37.4 & 5.4 & 1.8 & 0.1 & 23.7 & 0.7 & 0.6 & 0.1 \\
\hline $1^{4} \Pi$ & -14.980316 & 8.4 & 22.2 & 2.8 & 1.3 & 0.1 & 3.2 & 1.2 & 0.8 & 0.2 \\
\hline $2^{2} \Pi$ & -14.955116 & 29.8 & 42.0 & 4.1 & 0.8 & 0.1 & 26.7 & 0.3 & 0.3 & 0.1 \\
\hline $3^{2} \Pi$ & -14.921160 & 19.4 & 26.0 & 4.6 & 1.2 & 0.1 & 11.1 & 1.0 & 0.7 & 0.2 \\
\hline MAE & & 21.0 & 31.9 & 4.2 & 1.3 & 0.1 & 16.2 & 0.8 & 0.6 & 0.2 \\
\hline
\end{tabular}


The open-shell molecule $\mathrm{BeH}(R=1.3426 \AA)$ is used as another example. The lowest five $\Sigma^{+}$states and four $\Pi$ states are computed with FS-MPS and HS-MPS. In this case, both the spin projection $S_{z}$ and point group symmetry (Abelian subgroup $C_{2 v}$ ) are used. The results computed with a ROHF reference and the aug-cc-pVDZ basis ${ }^{60,61}$ are shown in Table IV. In this case, the theoretical bond dimensions of HS-MPS are $\{60,1610,9920,1610\}$ for $(K, N)=(64,5)$. However, it is seen that milli-Hartree accuracy can be achieved with a very small bond dimension such as 100 . The HS-MPS is more accurate than the FS-MPS with the same bond dimensions $D$ of 10,50 , and 100 . This remains the case until a very high accuracy of less than $0.1 m E_{h}$ is targeted. In the latter regime, FS-MPS becomes more advantageous as the situation $(K \gg N)$ is similar to that shown in Figure 6.

\section{CONCLUSIONS}

The traditional routes to approach FCI accuracy include utilizing all possible symmetries (antisymmetry, spin, and point group) to make the FCI space compact, truncating the FCI space based on excitation rank, or selecting important configurations based on perturbative estimates. TNS based methods provide a fundamentally different way to tackle the many-electron problem. By transforming the CI vector into a tensor, it allows to explore the low entanglement among different physical dimensions. Such low entanglement can either come from spatial or energetic locality. The redundancy of intermediate states can be reduced effectively through renormalization. The present paper answered a fundamental question: Can many-electron wavefunctions defined in a Hilbert space representation be approximated by products of smaller pieces as the TNS in the Fock-space case? The key for this problem is the introduction of prefix/suffix renormalization, which is a general tool to synthesize manybody states from smaller pieces in Hilbert space. The soobtained renormalized states have their own orbital supports that can be identified by the prefix or suffix, such that they can also be viewed as some special renormalized states in Fock space. Consequently, the problems of dealing with antisymmetry and computing matrix elements can be solved. The resulting HS-MPS Ansatz is highly flexible. By defining appropriate physical indices (nodes in the configuration graph) and coupling rules (interconnections among nodes), various configuration spaces can be represented, and the variational minimization of the energy in the linear space is converted to a minimization on the HS-MPS manifold (24). Several numerical examples have been studied to reveal the merits of HS-MPS and FS-MPS. The results are useful for guiding the design of new kinds of wavefunction Ansatz. In particular, the Ansatz obtained by limiting the maximal bond dimension but without restricting the physical indices and coupling rules has more flexibility than traditional truncated CI models. The important higher excitations can be sampled efficiently with moderate bond dimensions, in a very distinct way from, e.g., coupled-cluster theory. ${ }^{4}$ The usefulness of HS-MPS as a reference, to efficiently cover most of the strong correlations, and as a starting point for further including the residual dynamic correlation, deserves further exploration.
Hilbert-space formulations of tensor network states with other topologies, especially hierarchical tree (HT) structures,${ }^{62}$ may be useful in certain circumstances. The HT structure has seen application chemistry in the multilayer formulation of the multiconfiguration time-dependent Hartree theory $(\mathrm{ML}-\mathrm{MCTDH})^{63}$ in Fock space for identical particles. It can be related to tree tensor networks by the removal of the physical indices of all tensors except at the boundary of the tree. ${ }^{64-66}$ Its Hilbert-space version can be formulated with the help of the prefix/suffix renormalization. In particular, the spin-adapted HS-MPS proposed in Sec. III H can be viewed as a simple realization of such a HT structure.

\section{ACKNOWLEDGMENTS}

One of the authors (Z.L.) acknowledges helpful discussions with Sheng Guo, Sebastian Wounters, Qiming Sun, and Boxiao Zheng. This work was primarily supported by the US National Science Foundation, through the Award No. NSF CHE-1265277.

${ }^{1}$ P. A. M. Dirac, Proc. R. Soc. A 123, 714 (1929).

${ }^{2}$ T. Helgaker, P. Jørgensen, and J. Olsen, Molecular Electronic-Structure Theory (Wiley, 2013).

${ }^{3}$ I. Shavitt, Mol. Phys. 94, 3 (1998)

${ }^{4}$ I. Shavitt and R. J. Bartlett, Many-Body Methods in Chemistry and Physics. MBPT and Coupled-Cluster Theory (Cambridge University Press, 2009).

${ }^{5}$ S. Ten-no, Theor. Chem. Acc. 131, 1 (2012).

${ }^{6}$ L. Kong, F. A. Bischoff, and E. F. Valeev, Chem. Rev. 112, 75 (2011).

${ }^{7}$ C. Hättig, W. Klopper, A. Köhn, and D. P. Tew, Chem. Rev. 112, 4 (2011).

${ }^{8}$ G. K.-L. Chan, WIREs: Comput. Mol. Sci. 2, 907 (2012).

${ }^{9}$ R. Orús, Ann. Phys. 349, 117 (2014).

${ }^{10}$ S. Szalay, M. Pfeffer, V. Murg, G. Barcza, F. Verstraete, R. Schneider, and Ö. Legeza, Int. J. Quantum Chem. 115, 1342 (2015).

${ }^{11}$ S. R. White, Phys. Rev. Lett. 69, 2863 (1992).

${ }^{12}$ S. R. White, Phys. Rev. B 48, 10345 (1993).

${ }^{13}$ U. Schollwöck, Rev. Mod. Phys. 77, 259 (2005).

${ }^{14}$ U. Schollwöck, Ann. Phys. 326, 96 (2011).

${ }^{15}$ S. Östlund and S. Rommer, Phys. Rev. Lett. 75, 3537 (1995).

${ }^{16}$ S. R. White and R. L. Martin, J. Chem. Phys. 110, 4127 (1999).

${ }^{17}$ G. K.-L. Chan and M. Head-Gordon, J. Chem. Phys. 116, 4462 (2002).

${ }^{18}$ G. K.-L. Chan, J. Chem. Phys. 120, 3172 (2004).

${ }^{19}$ G. K.-L. Chan and S. Sharma, Annu. Rev. Phys. Chem. 62, 465 (2011).

${ }^{20}$ K. H. Marti and M. Reiher, Phys. Chem. Chem. Phys. 13, 6750 (2011).

${ }^{21}$ S. Wouters and D. Van Neck, Eur. Phys. J. D 68 (2014).

${ }^{22}$ Y. Kurashige, Mol. Phys. 112, 1485 (2014).

${ }^{23}$ R. Olivares-Amaya, W. Hu, N. Nakatani, S. Sharma, J. Yang, and G. K.-L. Chan, J. Chem. Phys. 142, 034102 (2015).

${ }^{24}$ Y. Kurashige, G. K.-L. Chan, and T. Yanai, Nat. Chem. 5, 660 (2013).

${ }^{25}$ S. Sharma, K. Sivalingam, F. Neese, and G. K.-L. Chan, Nat. Chem. 6, 927 (2014).

${ }^{26}$ E. Neuscamman, T. Yanai, and G. K.-L. Chan, Int. Rev. Phys. Chem. 29, 231 (2010).

${ }^{27}$ S. Sharma and G. K.-L. Chan, J. Chem. Phys. 141, 111101 (2014).

${ }^{28}$ M. Saitow, Y. Kurashige, and T. Yanai, J. Chem. Theory Comput. 11, 5120 (2015).

${ }^{29}$ S. Sharma and A. Alavi, J. Chem. Phys. 143, 102815 (2015).

${ }^{30}$ T. Yanai, Y. Kurashige, W. Mizukami, J. Chalupsk, T. N. Lan, and M. Saitow, Int. J. Quantum Chem. 115, 283 (2015).

${ }^{31}$ A. C. Hurley, J. Lennard-Jones, and J. A. Pople, Proc. R. Soc. A 220, 446 (1953).

${ }^{32}$ R. G. Parr, F. O. Ellison, and P. G. Lykos, J. Chem. Phys. 24, 1106 (1956).

${ }^{33}$ R. McWeeny, Proc. R. Soc. A 253, 242 (1959).

${ }^{34}$ W. Duch, J. Phys. A: Math. Gen. 18, 3283 (1985)

${ }^{35}$ R. Shepard, J. Phys. Chem. A 109, 11629 (2005).

${ }^{36}$ R. Shepard, J. Phys. Chem. A 110, 8880 (2006).

${ }^{37}$ R. Shepard, G. Gidofalvi, and S. R. Brozell, J. Chem. Phys. 141, 064105 (2014).

${ }^{38}$ R. Shepard, G. Gidofalvi, and S. R. Brozell, J. Chem. Phys. 141, 064106 (2014). 
${ }^{39}$ G. Gidofalvi, S. R. Brozell, and R. Shepard, Theor. Chem. Acc. 133, 1 (2014).

${ }^{40}$ M. B. Hastings, J. Stat. Mech.: Theory Exp. 2007, P08024.

${ }^{41}$ J. Eisert, M. Cramer, and M. B. Plenio, Rev. Mod. Phys. 82, 277 (2010).

${ }^{42}$ T. H. Dunning, Jr. and P. J. Hay, in Methods of Electronic Structure Theory (Modern Theoretical Chemistry), edited by H. F. Schaefer III (Springer, 1977), Vol. 3, Chap. 1, p. 1.

${ }^{43}$ J. Paldus, J. Chem. Phys. 61, 5321 (1974).

${ }^{44}$ I. Shavitt, Int. J. Quantum Chem. 12, 131 (1977).

${ }^{45}$ I. Shavitt, Int. J. Quantum Chem. 14, 5 (1978).

${ }^{46} \mathrm{H}$. Weyl, The Classical Groups: Their Invariants and Representations (Princeton University Press, 1939).

${ }^{47}$ M. Nakahara and T. Ohmi, Quantum Computing: From Linear Algebra to Physical Realizations (CRC Press, 2008).

${ }^{48}$ S. Sharma and G. K.-L. Chan, J. Chem. Phys. 136, 124121 (2012).

${ }^{49}$ D. J. Rowe and C. Ngo-Trong, Rev. Mod. Phys. 47, 471 (1975).

${ }^{50}$ F. Weinhold and E. B. Wilson, J. Chem. Phys. 46, 2752 (1967).

${ }^{51}$ D. Zgid and M. Nooijen, J. Chem. Phys. 128, 144115 (2008).

${ }^{52}$ T. Xiang, Phys. Rev. B 53, R10445 (1996).
${ }^{53}$ P.-A. Absil, R. Mahony, and R. Sepulchre, Optimization Algorithms on Matrix Manifolds (Princeton University Press, 2007).

${ }^{54}$ R. Pauncz, Spin Eigenfunctions: Construction and Use (Springer, 1979).

${ }^{55}$ S. Yabushita, Z. Zhang, and R. M. Pitzer, J. Phys. Chem. A 103, 5791 (1999).

${ }^{56}$ D. E. Knuth, The Art of Computer Programming, Volume 2: Seminumerical Algorithms, 3rd ed. (Addison-Wesley Professional, 1997).

${ }^{57}$ Q. Sun, PYSCF: Python module for quantum chemistry, 2016, https://github. com/sunqm/pyscf.

${ }^{58}$ T. H. Dunning, Jr., J. Chem. Phys. 53, 2823 (1970).

${ }^{59}$ C. W. Bauschlicher, Jr. and P. R. Taylor, J. Chem. Phys. 85, 2779 (1986).

${ }^{60}$ T. H. Dunning, Jr., J. Chem. Phys. 90, 1007 (1989).

${ }^{61}$ R. A. Kendall, T. H. Dunning, Jr., and R. J. Harrison, J. Chem. Phys. 96, 6796 (1992).

${ }^{62}$ Y.-Y. Shi, L.-M. Duan, and G. Vidal, Phys. Rev. A 74, 022320 (2006).

${ }^{63} \mathrm{H}$. Wang and M. Thoss, J. Chem. Phys. 131, 024114 (2009).

${ }^{64}$ V. Murg, F. Verstraete, Ö. Legeza, and R. Noack, Phys. Rev. B 82, 205105 (2010).

${ }^{65}$ N. Nakatani and G. K.-L. Chan, J. Chem. Phys. 138, 134113 (2013).

${ }^{66}$ V. Murg, F. Verstraete, R. Schneider, P. R. Nagy, and Ö. Legeza, J. Chem. Theory Comput. 11, 1027 (2015). 